\title{
Excited-state properties for extended systems: Efficient hybrid density functional methods.
}

\author{
Anna-Sophia Hehn, ${ }^{* \dagger}{ }^{\dagger}$ Beliz Sertcan, ${ }^{\dagger}$ Fabian Belleflamme, ${ }^{\dagger}$ Sergey K. Chulkov, ${ }^{\ddagger}$ \\ Matthew B. Watkins, ${ }^{\ddagger}$ and Jürg Hutter ${ }^{\dagger}$ \\ $\dagger$ Department of Chemistry, University of Zurich, Winterthurerstrasse 190, 8057 Zurich, \\ Switzerland \\ $\ddagger$ School of Mathematics and Physics, University of Lincoln, Brayford Pool, Lincoln \\ LN67TS, United Kingdom \\ E-mail: anna.hehn@chem.uzh.ch
}

\begin{abstract}
Time-dependent density functional theory has become state-of-the-art for describing photophysical and photochemical processes in extended materials due to its affordable cost. The inclusion of exact exchange was shown to be essential for the correct description of the long-range asymptotics of electronic interactions and thus a well-balanced description of valence, Rydberg and charge-transfer excitations. Several approaches for an efficient treatment of exact exchange have been established for the ground state, while implementations for excited-state properties are rare. Furthermore, the high computational costs required for excited-state properties in comparison to ground-state computations often hinder large-scale applications on periodic systems with hybrid functional accuracy. We therefore propose two approximate schemes for improving computational efficiency for the treatment of exact exchange. Within the auxiliary density matrix method (ADMM), exact exchange is estimated using a relatively small auxiliary basis and the introduced basis-set incompleteness error is compensated by an exchange density functional correction term. Benchmark results for a test set of 35 molecules demonstrate that the mean absolute error introduced by ADMM is smaller than $0.2 \mathrm{pm}$ for excited-state bond lengths and in the range of $0.02-0.06 \mathrm{eV}$ for vertical excitation, adiabatic excitation and fluorescence energies. Computational timings for
\end{abstract}


a series of covalent-organic frameworks demonstrate that a speed-up of at least one order of magnitude can be achieved for ES geometry optimizations in comparison to conventional hybrid functionals. The second method is to use a semi-empirical tight binding approximation for both Coulomb and exchange contributions to the excitedstate kernel. This simplified Tamm-Dancoff approximation (sTDA) achieves an accuracy comparable to approximated hybrid density functional theory when referring to highly accurate coupled-cluster reference data. We find that excited-state bond lengths deviate by $1.1 \mathrm{pm}$ on average and mean absolute errors in vertical excitation, adiabatic excitation and fluorescence energies are in the range of $0.2-0.5 \mathrm{eV}$. In comparison to ADMM-approximated hybrid functional theory, sTDA accelerates the computation of broad-band excitation spectra by one order of magnitude, suggesting its potential use for large-scale screening purposes. 


\section{Introduction}

The description of excited states in large extended systems using quantum-mechanical approaches is still a challenge for theoretical spectroscopy. ${ }^{1}$ In contrast to the electronic ground state, the quest of finding an appropriate model with well-balanced accuracy-cost ratio for the excited state is complicated by various electronic states of different nature having to be described simultaneously. For instance, this renders the parametrization of transferable classical force fields for excited states an almost impractical task. The cost of highly accurate wave-function methods scales prohibitively with system size making routine large scale applications impossible. When aiming for a robust and efficient tool set for the treatment of extended periodic systems, density functional theory and semi-empirical tight binding approaches therefore represent a suitable compromise. While time-dependent density functional theory (TDDFT) has been established as efficient and broadly applicable for the treatment of excited states, ${ }^{2,3}$ there has also been a recent revival of semi-empirical approaches to extend treatable system sizes from hundreds to thousands of atoms. ${ }^{4}$ Both the re-emergence of semi-empirical methods as well as the continuing improvement of density functional approximations are among the most important future developments in computational chemistry. ${ }^{5}$ The correct description of exact exchange has proven to be crucial for an adequate treatment of excited states in TDDFT. It has a much greater influence on the geometrical displacement upon excitation, and thus on transition energies and spectra, than the exchange-correlation functional. ${ }^{6}$ Benchmarks on molecular systems, including radicals and ions, suggest including $30-40 \%$ exact exchange to obtain reliable spectroscopic data. ${ }^{6}$ Straight-forward evaluation of the two-electron exact exchange integrals leads to a formal scaling of $N^{4}$ with system size $N$ for localized basis sets, emphasizing the need for more cost-efficient approximation schemes. An overview of recent developments and the various existing algorithms can e.g. be found in Ref. ${ }^{7}$ One approach is the auxiliary density matrix method (ADMM), ${ }^{8}$ where the gain in efficiency is achieved by evaluating a model exact exchange energy within a relatively small auxiliary basis augmented by a correction term based on a local exchange functional. The assumption that the basis-set incompleteness error can be corrected in terms of a cost-

efficient density functional correction term was shown to be well-founded ${ }^{7}$ and benchmarks including liquids, solids as well as proteins in solution with up to 3000 atoms revealed that memory requirements and computational efficiency are improved by at least one order of magnitude. ${ }^{8}$ Kumar et al. showed that ADMM achieves good accuracy for ground- and excited-state energies, even though rather large errors were found for polarizabilities and hyperpolarizabilities. ${ }^{9}$ The least complex variant of ADMM is based on a simple least-squares fitting projection of molecular orbitals onto the smaller auxiliary basis. More sophisticated 
ADMM variants have been proposed including density matrix purification and projections enforcing orthogonality or charge constraints. ${ }^{7}$ Comparing amongst the developed ADMM variants, additional constraints as well as an adequate choice of the exchange functional for the correction term were shown to further improve accuracy for total energies while retaining a comparable efficiency. With respect to other sophisticated exact exchange algorithms ${ }^{10}$ including the pair-atomic resolution-of-the-identity method (PARI-K) ${ }^{11,12}$ and the chain-ofspheres algorithm (COSX), ${ }^{13-16}$ total as well as orbital, reaction and atomization energies were found to be one to three orders of magnitude less accurate. However, ADMM achieves a computational efficiency comparable to the cost required for density fitting Coulomb algorithms. ADMM was thus classified as promising model with an "impressive speedup", encouraging further development to improve accuracy and calling for careful optimization of the required auxiliary basis sets. ${ }^{10}$

For even larger system sizes of several thousands of atoms it is necessary to go from $a b$ initio excited-state calculations towards semi-empirical tight binding approximations. In recent years, a class of tight binding approaches was suggested by Grimme et al., ${ }^{4}$ based on the idea of reducing the computational effort of electron repulsion integrals while retaining an adequate description of the physics of electronic interactions. More specifically, Coulomb and exchange contributions are approximated using an electron repulsion operator that captures the correct long-range $1 / R$ asymptotics by construction ${ }^{17-19}$ but allows for a short range empirical parametrization. It was shown that this choice provides a balanced description of valence, charge-transfer as well as Rydberg states. The developed methods were dubbed simplified TDDFT (sTDDFT) ${ }^{20}$ or simplified Tamm-Dancoff approximation (sTDA $)^{21,22}$ corresponding to the related ab initio electronic structure method and were extended to treat e.g. spin-flip excitations ${ }^{23-26}$ or to be combined with the idea of range separation. ${ }^{27}$ In contrast to other common tight binding approaches like DFTB, ${ }^{28}$ sTDA and sTDDFT involve only a limited number of global parameters which enables their straightforward application over the whole periodic table. ${ }^{29}$ Benchmark results demonstrated that the simplified approaches achieve computational savings of at least two orders of magnitude while the loss in accuracy is minor with an average deviation of $0.2-0.3 \mathrm{eV}$ for excitation energies when being compared to conventional TDDFT or experiment. If based on a semiempirical ground state reference, sTDA errors were found to be slightly larger with mean absolute deviations in the range of $0.3-0.5 \mathrm{eV}$. Most importantly, accuracy was found to be consistent for both the low-energy valence as well as the high-energy Rydberg transitions. ${ }^{21,22}$ Simplified approaches were also suggested for the Bethe-Salpeter equation (BSE) and the GW approximation. ${ }^{30}$ These methods provided GW ionization potentials within the GW100 test set differing only by $0.2 \mathrm{eV}$ while enabling cubic scaling with system size. Deviations 
in sBSE excitation energies amount up to $0.5 \mathrm{eV}$ and are thus in an error range comparable to sTDA and sTDDFT. ${ }^{30}$ A closely related TDDFT + TB approach has been suggested by Visscher et al. which relies on the same monopole approximation for the electron repulsion integrals, but is - in contrast to sTDA and sTDDFT - not designed for hybrid, but pure density functionals. ${ }^{31,32}$

The mentioned advantages of ADMM and sTDA regarding their physically correct description of exact exchange qualify both approaches as promising for an efficient calculation of excited-state properties. Excited-state gradients within the framework of linear response TDDFT were pioneered for molecular systems by Van Caillie and Amos ${ }^{33,34}$ as well as Furche and Ahlrichs. ${ }^{35,56}$ This work was extended to periodic systems and plane wave basis sets. ${ }^{36}$ Furthermore, TDDFT excited-state properties for exact exchange have been extended to global and local hybrid functionals ${ }^{37,38}$ as well as range separation. ${ }^{39}$ Implementations are also available for tight binding approaches including long-range corrections. ${ }^{40}$ However, algorithms are most often restricted to molecular systems. We implemented sTDA and ADMMapproximated hybrid density functional excited-state gradients based on the Gaussian and plane wave (GPW) framework within the CP2K program package. ${ }^{41-43}$ The GPW methods allow for a natural extension of algorithms to periodic boundary conditions and thus enable the treatment of extended systems. The implementation is based on earlier works of Iannuzzi et al. ${ }^{44}$ and Strand et al. ${ }^{45}$ already featuring the calculation of ADMM excitation energies for model systems of up to 1000 atoms. ${ }^{46}$ As outlined in section 2.1, the CP2K implementation is based on the Sternheimer equations, thus depending solely on the occupied molecular and atomic orbital space. ? ' Such a formulation requires e.g. adjustments in the formulation of the ADMM equations and the sTDA eigenvalue problem, see sections 2.2 and 2.3. Going from molecular to periodic systems also requires Coulomb interactions to be treated using Ewald summation techniques and the minimum image approximation to capture exact exchange, see section 2.4. We tested our excited-state gradient implementations using a molecular benchmark set of Jacquemin et al. ${ }^{47}$ which is one of the state-of-the-art test sets for excited-state properties. ADMM-approximated hybrid functional and semi-empirical sTDA kernels are compared to conventional hybrid functional TDDFT exploiting the therein provided EOM-CCSD geometries and ES data. Finally, in section 3.2, the computational efficiency of the proposed algorithms is demonstrated by applications on porous covalent organic framework (COF) materials taken from the CURATED COFs database. ${ }^{48,49}$ 


\section{Theoretical background}

\subsection{The Tamm-Dancoff approximation}

Within the Tamm-Dancoff approximation, ${ }^{50}$ the excitation energy $\Omega$ and corresponding excited-state eigenvectors $\mathbf{X}$ for each excited state are defined in terms of the variational Lagrangian $G$,

$$
\begin{aligned}
G\left[\mathbf{X}, \mathbf{C}, \Omega, \overline{\mathbf{W}}^{\mathrm{x}}\right] & =\sum_{\kappa k \sigma} X_{\kappa k \sigma}^{\mathrm{T}} \sum_{\lambda l}\left[F_{\kappa \lambda \sigma} \delta_{k l}-F_{k l \sigma} S_{\kappa \lambda}\right] X_{\lambda l \sigma}+\sum_{\kappa \lambda \sigma} D_{\kappa \lambda \sigma}^{\mathrm{x}} K_{\kappa \lambda \sigma}\left[\mathbf{D}^{\mathrm{x}}\right] \\
& -\sum_{\kappa \lambda k l \sigma} \Omega\left(X_{\kappa k \sigma}^{\mathrm{T}} S_{\kappa \lambda} X_{\lambda l \sigma}-\delta_{k l}\right) \\
& -\sum_{k l \sigma}\left(\bar{W}_{k l \sigma}^{\mathrm{x}}\right)^{\mathrm{T}} \sum_{\kappa \lambda} \frac{1}{2}\left(C_{\kappa k \sigma}^{\mathrm{T}} S_{\kappa \lambda} X_{\lambda l \sigma}+X_{\kappa k \sigma}^{\mathrm{T}} S_{\kappa \lambda} C_{\lambda l \sigma}\right),
\end{aligned}
$$

implying the stationary conditions

$$
\begin{aligned}
& \frac{\partial G}{\partial \mathbf{X}}=0 \rightarrow \sum_{\kappa k}\left[F_{\mu \kappa \sigma} \delta_{i k}-F_{i k \sigma} S_{\mu \kappa}\right] X_{\kappa k \sigma}+\sum_{\kappa \lambda} Q_{\mu \kappa}^{\mathrm{T}} K_{\kappa \lambda \sigma}\left[\mathbf{D}^{\mathrm{x}}\right] C_{\lambda i \sigma}=\sum_{\kappa} \Omega S_{\mu \kappa} X_{\kappa i \sigma}, \\
& \frac{\partial G}{\partial \Omega}=0 \rightarrow \sum_{\kappa \lambda \sigma} X_{\kappa i \sigma}^{\mathrm{T}} S_{\kappa \lambda} X_{\lambda j \sigma}=\delta_{i j} \\
& \frac{\partial G}{\partial \overline{\mathbf{W}}^{\mathrm{x}}}=0 \rightarrow \sum_{\kappa \lambda k l \sigma}\left(C_{\kappa k \sigma}^{\mathrm{T}} S_{\kappa \lambda} X_{\lambda l \sigma}+X_{\kappa k \sigma}^{\mathrm{T}} S_{\kappa \lambda} C_{\lambda l \sigma}\right)=0
\end{aligned}
$$

Eq. (2) represents a hermitian eigenvalue problem which is for molecular orbital (MO) based formulations solved under the constraint that the excited-state eigenvectors remain orthonormalized (Eq. (3)). ${ }^{51}$ The Lagrange multiplier introducing the normalization constraint of Eq. (3) is thereby chosen to be equal to the excitation energy $\Omega$ according to the canonical gauge. In an atomic orbital (AO)-based formalism relying solely on occupied $\mathrm{MOs},\{i, j, k, l, \ldots\}$, and AOs, $\{\mu, \nu, \kappa, \lambda, \ldots\}$, it furthermore has to be ensured that the excited-state eigenvector $\mathbf{X}$ is orthogonal to the ground-state (GS) MO coefficients $\mathbf{C}$ (Eq. (4)), a constraint introduced in the Lagrangian $G$ via the Lagrange multiplier $\overline{\mathbf{W}}^{\mathrm{x}}$,

$$
X_{i j \sigma}=\sum_{\kappa \lambda} C_{\kappa i \sigma}^{\mathrm{T}} S_{\kappa \lambda} X_{\lambda j \sigma}=0
$$

By taking the derivative of $G$ with respect to the excited-state eigenvectors $\mathbf{X}$ and projecting onto the occupied MO coefficients $\mathbf{C}$, it can be shown that this second normalization constraint is representing a projection of the kernel contributions onto the virtual space, already 
inserted in the eigenvalue problem of Eq. (2) in terms of the projection operator $\mathbf{Q}$,

$$
\begin{aligned}
& Q_{\mu \nu \sigma}=\delta_{\mu \nu}-\sum_{\kappa k} C_{\mu k \sigma} C_{\kappa k \sigma}^{\mathrm{T}} S_{\kappa \nu}, \\
& Q_{\mu \nu \sigma}^{\mathrm{T}}=\delta_{\nu \mu}-\sum_{\kappa k} S_{\nu \kappa} C_{\kappa k \sigma} C_{\mu k \sigma}^{\mathrm{T}} .
\end{aligned}
$$

Both constraints are ensured in the TDDFT module of CP2K by explicitly orthonormalizing the ES eigenvectors as well as by orthogonalizing the ES eigenvectors with respect to the MO coefficients at each step of the Davidson algorithm. Eq. (5) implies the transformation rules from $\mathrm{AO}$ to $\mathrm{MO}$ basis and vice versa, which are given for vectors $\mathbf{V}$ and matrices $\mathbf{M}$ in general as

$$
\begin{aligned}
V_{\mu i \sigma}^{\mathrm{AO}} & =\sum_{k} C_{\mu k \sigma} V_{k i \sigma}^{\mathrm{MO}}, \\
V_{i j \sigma}^{\mathrm{MO}} & =\sum_{\kappa \lambda} C_{\kappa i \sigma}^{\mathrm{T}} S_{\kappa \lambda} V_{\lambda j \sigma}^{\mathrm{AO}}, \\
M_{\mu \nu \sigma}^{\mathrm{AO}} & =\sum_{\kappa \lambda k l} S_{\mu \kappa} C_{\kappa k \sigma}^{\mathrm{T}} M_{k l \sigma}^{\mathrm{MO}} C_{l \lambda \sigma} S_{\lambda \nu}, \\
M_{i j \sigma}^{\mathrm{MO}} & =\sum_{\kappa \lambda} C_{\kappa i \sigma}^{\mathrm{T}} M_{\kappa \lambda \sigma}^{\mathrm{AO}} C_{\lambda j \sigma},
\end{aligned}
$$

with the overlap matrix $\mathbf{S}$ being defined in terms of the $\operatorname{AOs} \varphi_{\mu}(\mathbf{r})$,

$$
S_{\mu \nu}=\int \varphi_{\mu}(\mathbf{r}) \varphi_{\nu}(\mathbf{r}) \mathrm{d} \mathbf{r}
$$

Depending on the applied density functional, the eigenvalue problem of Eq. (2) comprises one-electron $\mathbf{h}$, Coulomb $\mathbf{J}$ and exact exchange $\mathbf{K}^{\mathrm{Ex}}$ contributions as well as contributions due to the exchange-correlation $(\mathrm{XC})$ potential $V_{\sigma}^{\mathrm{xC}}(\mathbf{r})$ or kernel $f_{\sigma \sigma^{\prime}}^{\mathrm{xC}}\left(\mathbf{r}, \mathbf{r}^{\prime}\right)$, with the Kohn-Sham matrix $\mathbf{F}$ and the kernel matrix $\mathbf{K}$ being defined as

$$
\begin{aligned}
F_{\mu \nu \sigma}[\mathbf{D}] & =h_{\mu \nu}+J_{\mu \nu \sigma}[\mathbf{D}]-a_{\mathrm{EX}} K_{\mu \nu \sigma}^{\mathrm{Ex}}[\mathbf{D}]+V_{\mu \nu \sigma}^{\mathrm{xC}}, \\
& =h_{\mu \nu}+\sum_{\kappa \lambda \sigma^{\prime}} D_{\kappa \lambda \sigma^{\prime}}\left[(\mu \nu \mid \kappa \lambda)-a_{\mathrm{Ex}} \delta_{\sigma \sigma^{\prime}}(\mu \kappa \mid \nu \lambda)\right]+V_{\mu \nu \sigma}^{\mathrm{xC}}, \\
K_{\mu \nu \sigma}\left[\mathbf{D}^{\mathrm{X}}\right] & =J_{\mu \nu \sigma}\left[\mathbf{D}^{\mathrm{X}}\right]-a_{\mathrm{EX}} K_{\mu \nu \sigma}^{\mathrm{Ex}}\left[\mathbf{D}^{\mathrm{X}}\right]+\sum_{\kappa \lambda \sigma^{\prime}} f_{\mu \nu \sigma, \kappa \lambda \sigma^{\prime}}^{\mathrm{XC}} D_{\kappa \lambda \sigma^{\prime}}^{\mathrm{X}}, \\
& =\sum_{\kappa \lambda \sigma^{\prime}} D_{\kappa \lambda \sigma^{\prime}}^{\mathrm{x}}\left[(\mu \nu \mid \kappa \lambda)-a_{\mathrm{EX}} \delta_{\sigma \sigma^{\prime}}(\mu \kappa \mid \nu \lambda)+f_{\mu \nu \sigma, \kappa \lambda \sigma^{\prime}}^{\mathrm{XC}}\right] .
\end{aligned}
$$


$a_{\mathrm{Ex}}$ is a global parameter to scale the amount of exact exchange and the two-electron repulsion integrals are defined as

$$
(\mu \nu \mid \kappa \lambda)=\int \mathrm{d} \mathbf{r} \int \mathrm{d} \mathbf{r}^{\prime} \varphi_{\mu}(\mathbf{r}) \varphi_{\nu}(\mathbf{r}) \frac{1}{\left|\mathbf{r}-\mathbf{r}^{\prime}\right|} \varphi_{\kappa}\left(\mathbf{r}^{\prime}\right) \varphi_{\lambda}\left(\mathbf{r}^{\prime}\right)
$$

The corresponding density matrices $\mathbf{D}$ and $\mathbf{D}^{\mathrm{x}}$ are defined based on the MO coefficients $\mathbf{C}$ implying symmetrization,

$$
\begin{aligned}
& D_{\mu \nu \sigma}=\sum_{k} C_{\mu k \sigma} C_{\nu k \sigma}^{\mathrm{T}}, \\
& D_{\mu \nu \sigma}^{\mathrm{x}}=\frac{1}{2} \sum_{k}\left(X_{\mu k \sigma} C_{\nu k \sigma}^{\mathrm{T}}+C_{\mu k \sigma} X_{\nu k \sigma}^{\mathrm{T}}\right) .
\end{aligned}
$$

Symmetrization is necessary to ensure that the linear response density is real. Furthermore, within the implementation for periodic systems using a $\Gamma$-point only description, we can assume real wave functions. The basis functions and MOs are periodically replicated, integrals are over the computational unit cell, and all Coulomb terms evaluated using Ewald sums.

\subsection{Exact exchange using the Auxiliary Density Matrix Method $(\mathrm{ADMM})$}

The basic idea of $\mathrm{ADMM}^{7,52}$ is to introduce a small and rapidly decaying auxiliary density matrix $\hat{\mathbf{D}}$ to speed up the calculation of the exact Hartree-Fock exchange matrix, $\mathbf{K}^{\mathrm{Ex}}$, with the latter being evaluated within a smaller auxiliary basis, $\{\hat{\mu}, \hat{\nu}, \hat{\kappa}, \hat{\lambda}, \ldots\}$. The total exchange energy contribution to the ES energy is thus approximated by a model term, $E^{\mathrm{Ex}}[\hat{\mathbf{D}}]$, and to compensate the so-introduced basis-set incompleteness error, a correction term based on a local density functional $E^{\mathrm{GGA}}$ is added,

$$
E^{\mathrm{EX}}[\mathbf{D}] \approx E^{\mathrm{EX}}[\hat{\mathbf{D}}]+\left[E^{\mathrm{GGA}}[\mathbf{D}]-E^{\mathrm{GGA}}[\hat{\mathbf{D}}]\right]
$$

Different approaches have been developed to obtain the auxiliary density matrix $\hat{\mathbf{D}}$ and corresponding MO coefficients $\hat{\mathbf{C}}$, e.g. by minimizing solely the square difference between the occupied orbital and auxiliary basis functions or by adding an orthonormality constraint to the fitting procedure. We will restrict the discussion to the ADMM2 variant, which is often also dubbed non-purified wave function fitting and which can be expressed in terms of the projection matrix $\hat{\mathbf{U}}$. For the sake of convenience, we will nevertheless refer to ADMM2approximated results in the following by the general acronym ADMM. Auxiliary basis functions and corresponding auxiliary matrices are indicated as $\hat{\mu}$ and $\hat{\mathbf{M}}$. The projection matrix 
$\hat{\mathbf{U}}$ is obtained according to

$$
\begin{aligned}
& \hat{\mathbf{U}}=\hat{\mathbf{S}}^{-1} \hat{\mathbf{V}}, \\
& \hat{\mathbf{D}}=\hat{\mathbf{C}} \hat{\mathbf{C}}^{\mathrm{T}}=\hat{\mathbf{U} D \mathbf{U}^{\mathrm{T}},} \\
& \hat{\mathbf{C}}=\hat{\mathbf{U}} \mathbf{C},
\end{aligned}
$$

based on the overlap matrices $\hat{\mathbf{S}}$ and $\hat{\mathbf{V}}$ of the auxiliary and mixed auxiliary-primary basis,

$$
\begin{aligned}
& \hat{S}_{\hat{\mu} \hat{\nu}}=\int \hat{\varphi}_{\hat{\mu}}(\mathbf{r}) \hat{\varphi}_{\hat{\nu}}(\mathbf{r}) \mathrm{d} \mathbf{r}, \\
& \hat{V}_{\hat{\mu} \nu}=\int \hat{\varphi}_{\hat{\mu}}(\mathbf{r}) \varphi_{\nu}(\mathbf{r}) \mathrm{d} \mathbf{r} .
\end{aligned}
$$

The exact exchange matrix $\mathbf{K}^{\mathrm{Ex}}$ (Eq. (14)) is thus approximated within ADMM as

$$
K_{\mu \nu \sigma}^{\mathrm{EX}}\left[\mathbf{D}^{\mathrm{X}}\right] \approx K_{\mu \nu \sigma}^{\mathrm{EX}, \mathrm{ADMM}}\left[\mathbf{D}^{\mathrm{X}}\right]=\sum_{\hat{\mu} \hat{\nu}} \hat{U}_{\hat{\mu} \mu}^{\mathrm{T}} \hat{K}_{\hat{\mu} \hat{\nu} \sigma}^{\mathrm{EX}}\left[\hat{\mathbf{D}}^{\mathrm{X}}\right] \hat{U}_{\hat{\nu} \nu}+K_{\mu \nu \sigma}^{\mathrm{GGA}, \mathrm{ES}},
$$

with the local GGA exchange density functional correction term

$$
K_{\mu \nu \sigma}^{\mathrm{GGA}, \mathrm{ES}}=\sum_{\kappa \lambda \sigma^{\prime}} f_{\mu \nu \sigma, \kappa \lambda \sigma^{\prime}}^{\mathrm{GGA}}[\mathbf{D}] D_{\kappa \lambda \sigma^{\prime}}^{\mathrm{x}}-\sum_{\hat{\mu} \hat{\nu}} \sum_{\hat{\kappa} \hat{\lambda} \sigma^{\prime}} \hat{U}_{\hat{\mu} \mu}^{\mathrm{T}} f_{\hat{\mu} \hat{\nu} \sigma, \hat{\kappa} \hat{\lambda} \sigma^{\prime}}^{\mathrm{GGA}}[\hat{\mathbf{D}}] \hat{U}_{\hat{\nu} \nu} \sum_{\kappa \lambda} \hat{U}_{\hat{\kappa} \kappa} D_{\kappa \lambda \sigma^{\prime}}^{\mathrm{X}} \hat{U}_{\hat{\lambda} \lambda}^{\mathrm{T}} .
$$

If relying also on an ADMM-approximated GS reference, exchange contributions to the KS matrix $\mathbf{F}$ of Eq. (13) imply an analogous correction with the second term of Eq. (24) now depending on the XC potential,

$$
K_{\mu \nu \sigma}^{\mathrm{GGA}, \mathrm{GS}}=V_{\mu \nu \sigma}^{\mathrm{GGA}}[\mathbf{D}]-\sum_{\hat{\mu} \hat{\nu}} \hat{U}_{\hat{\mu} \mu}^{\mathrm{T}} V_{\hat{\mu} \hat{\nu} \sigma}^{\mathrm{GGA}}[\hat{\mathbf{D}}] \hat{U}_{\hat{\nu} \nu}
$$

\subsection{Semi-empirical Coulomb and Exchange contributions within the simplified Tamm-Dancoff approximation (sTDA)}

In contrast to conventional TDA, sTDA ${ }^{21}$ neglects all contributions due to the exchangecorrelation kernel and approximates the remaining two-electron repulsion integrals based on the semi-empirical Mataga-Nishimoto-Ohno-Klopman operator $\gamma(A, B) \cdot{ }^{17-19}$ The simplified 
kernel contribution to Eq. (2) is given as

$$
\begin{aligned}
\sum_{\lambda} K_{\mu \lambda \sigma}^{\mathrm{sTDA}}\left[\mathbf{D}^{\mathrm{x}}\right] C_{\lambda i \sigma} & =\sum_{A B}(1-s) \gamma^{\mathrm{J}}(A, B) \tilde{\widetilde{C}}_{\mu i \sigma}^{B} \sum_{\lambda_{\mathrm{A}} l \sigma^{\prime}} \tilde{C}_{\lambda_{\mathrm{A}} l \sigma^{\prime}} \tilde{X}_{\lambda_{\mathrm{A}} l \sigma^{\prime}} \\
& -\sum_{A B} \gamma^{\mathrm{EX}}(A, B) \sum_{l} \tilde{X}_{\mu l \sigma}^{B} q_{i l \sigma}^{A}
\end{aligned}
$$

with $\gamma(A, B)$ describing either Coulomb (J) or exchange (EX) interactions depending on the interatomic distance $R_{A B}$ of atoms $A$ and $B$,

$$
\begin{aligned}
\gamma^{J}(A, B) & =\left(\frac{1}{\left(R_{A B}\right)^{\alpha}+\eta^{-\alpha}}\right)^{1 / \alpha}, \\
\gamma^{\mathrm{EX}}(A, B) & =\left(\frac{1}{\left(R_{A B}\right)^{\beta}+\left(a_{\mathrm{EX}} \eta\right)^{-\beta}}\right)^{1 / \beta} .
\end{aligned}
$$

Note that the nomenclature classifying Eqs. (28) and (29) as exchange- or Coulomb-like interaction operators differs from the original paper ${ }^{21}$ to match with the definitions for ADMM. The parameter $s$ is equal to -1 for singlet closed-shell wave functions, for triplet closed-shell it is set to $s=1$ and for open-shell wave-functions to $s=0$. Four different global parameters are included: the chemical hardness $\eta$ which is specified for each element according to Ref., ${ }^{53}$ powers of $\alpha$ and $\beta$ allowing to modify the distance dependence of $\gamma$ independently for either Coulomb or exchange interactions, and, analogously to conventional TDA, a Fock-exchange mixing parameter $a_{\mathrm{Ex}}$. The latter can be chosen freely, it was adjusted for molecular systems and global hybrids ${ }^{21}$ and in this case shows best performance for $a_{\mathrm{Ex}}=0.5 \cdot{ }^{22}$ Furthermore, we chose to set $\gamma^{\mathrm{EX}}(A, B)$ to zero if $a_{\mathrm{Ex}}=0$. The transition density charge $\mathbf{q}^{A}$ is defined as

$$
q_{i j \sigma}^{A}=\sum_{\kappa_{A}} \tilde{C}_{\kappa_{A} i \sigma}^{\mathrm{T}} \tilde{C}_{\kappa_{A j \sigma}}
$$

with the sum over $\kappa_{A}$ running over all atomic orbitals located at atom $A$. $\tilde{\mathbf{C}}$ or $\tilde{\mathbf{X}}$ are Löwdin transformed MO coefficients or excitation vectors and $\widetilde{\mathbf{C}}$ and $\tilde{\mathbf{X}}$ related doubly contracted intermediates,

$$
\begin{aligned}
& \tilde{C}_{\mu i \sigma}=\sum_{\eta} S_{\mu \eta}^{1 / 2} C_{\eta i \sigma}, \\
& \widetilde{C}_{\mu i \sigma}^{A}=\sum_{\eta_{A}} S_{\mu \eta_{A}}^{1 / 2} \tilde{C}_{\eta_{A} i \sigma} .
\end{aligned}
$$




\subsection{Periodic boundary conditions}

When generalizing the methods to account for periodic boundary conditions (PBC), an adequate description of the long-range Coulomb forces using Ewald summation techniques is required to ensure convergence of the slowly decaying potential at large distances. ${ }^{54}$ In the case of sTDA, the Coulomb operator is therefore split into a semi-empirical short-range and an exact long-range contribution,

$$
\gamma^{\mathrm{J}}(A, B) \stackrel{\mathrm{PBC}}{\longrightarrow} \gamma_{\mathrm{PBC}}^{\mathrm{J}}(A, B)+\frac{1}{R_{A B}}
$$

implying that the semi-empirical electron repulsion operator $\gamma_{\mathrm{PBC}}^{\mathrm{J}}$ for the short-range contribution is cut off at an atom-specific radius $R_{\text {cut }}$,

$$
\begin{array}{ll}
\gamma_{\mathrm{PBC}}^{\mathrm{J}}(A, B)=0 & \text { if } R_{A B}>R_{\text {cut }}, \\
\gamma_{\mathrm{PBC}}^{\mathrm{J}}(A, B)=\eta & \text { if } R_{A B}<10^{-6} \text { a.u. }, \\
\gamma_{\mathrm{PBC}}^{\mathrm{J}}(A, B)=\gamma^{\mathrm{J}}(A, B) & \text { if } 10^{-6} \text { a.u. } \leq R_{A B}<\left(R_{\text {cut }}-R_{\text {smooth }}\right), \\
\gamma_{\mathrm{PBC}}^{\mathrm{J}}(A, B)=f(\bar{R}) \gamma^{\mathrm{J}}(A, B)-\frac{f(\bar{R})}{R_{A B}} & \text { if }\left(R_{\text {cut }}-R_{\text {smooth }}\right) \leq R_{A B} \leq R_{\text {cut }} .
\end{array}
$$

$R_{\text {cut }}$ is defined according to the cutoff radius of the atomic basis functions and the function $f(\bar{R})$ smoothes the potential around $R_{\text {cut }}$ and is chosen such that both the first and second derivative of $\gamma^{\mathrm{J}}$ vanish at the cut-off borders,

$$
\begin{aligned}
f(\bar{R}) & =-6 \bar{R}^{5}+15 \bar{R}^{4}-10 \bar{R}^{3}+1 \\
\bar{R} & =R_{A B}-\left(R_{\text {cut }}-R_{\text {smooth }}\right) / R_{\text {smooth }}
\end{aligned}
$$

The long-range contribution $\frac{1}{R_{A B}}$ is calculated using the smooth particle mesh Ewald method as implemented for tight binding approaches in $\mathrm{CP} 2 \mathrm{~K},{ }^{55}$ scaling as $N \log N$ with system size $N$. Exchange interactions based on $\gamma^{\mathrm{EX}}$ are treated by implying the minimum image convention, thus restricting the sum over $A B$ to neighbors within the unit cell.

\subsection{The excited-state Lagrangian in the Tamm-Dancoff approxima- tion}

Nuclear gradients for the excited state are state-of-the-art in many program codes, so that the general procedure will only be outlined in short to discuss modifications that are necessary for ADMM and sTDA kernels. To avoid the computational cost of calculating the first-order response of the $\mathrm{MO}$ coefficients and to take into account the geometry dependence of the 
AOs, the Lagrangian of Eq. (1) needs to be extended by means of two additional constraints and thus is given as,

$$
\begin{aligned}
L\left[\mathbf{X}, \mathbf{C}, \Omega, \overline{\mathbf{W}}^{\mathrm{x}}, \overline{\mathbf{Z}}, \overline{\mathbf{W}}^{\mathrm{C}}\right] & =G\left[\mathbf{X}, \mathbf{C}, \Omega, \overline{\mathbf{W}}^{\mathrm{x}}\right]-\sum_{k l \sigma}\left(\bar{W}_{k l \sigma}^{\mathrm{C}}\right)^{\mathrm{T}}\left(S_{k l \sigma}-\delta_{k l}\right) \\
& +\sum_{\kappa k \sigma}\left(\bar{Z}_{\kappa k \sigma}\right)^{\mathrm{T}} \sum_{\lambda}\left(F_{\kappa \lambda \sigma} C_{\lambda k \sigma}-S_{\kappa \lambda} C_{\lambda k \sigma} \varepsilon_{k \sigma}\right) .
\end{aligned}
$$

The first additional constraint ensures the stationarity of the GS Kohn-Sham (KS) equations, the Brillouin condition, which can be rearranged using the definition of $\mathbf{Q}$ to emphasize the equivalence with MO-based formulations,

$$
\sum_{i a \sigma} \bar{Z}_{i a \sigma} F_{a i \sigma}=\sum_{i \kappa \lambda \sigma} \bar{Z}_{i \lambda \sigma} Q_{\kappa \lambda \sigma}^{\mathrm{T}} F_{\kappa i \sigma}=\sum_{i \kappa \lambda \sigma} \bar{Z}_{i \lambda \sigma}\left(F_{\lambda \kappa \sigma} C_{\kappa i \sigma}-S_{\lambda \kappa} C_{\kappa i \sigma} \varepsilon_{i \sigma}\right)
$$

Note that only the virtual-occupied part of the $\mathrm{Z}$ vector $\overline{\mathbf{Z}}$ is taken into account and that the occupied-occupied part is implied to be zero,

$$
\bar{Z}_{i j \sigma}=\sum_{\kappa \lambda} \bar{Z}_{i \lambda \sigma} S_{\lambda \kappa} C_{\kappa j \sigma}=0
$$

The second additional constraint ensures the orthogonality of the occupied MOs and, in analogy to $\overline{\mathbf{W}}^{\mathrm{x}}$ and $\overline{\mathbf{Z}}$, the hereby introduced Lagrange multiplier $\overline{\mathbf{W}}^{\mathrm{c}}$ is assumed to be symmetric. $\overline{\mathbf{W}}^{\mathrm{C}}$ and $\overline{\mathbf{Z}}$ are determined by taking the derivative of $L$ with respect to the MO coefficients $\mathbf{C}$ and projecting onto either the virtual or occupied orbital space,

$$
\frac{\partial L}{\partial \mathbf{C}} \mathbf{C}=0 \rightarrow \overline{\mathbf{W}}^{\mathrm{C}} ; \frac{\partial L}{\partial \mathbf{C}} \mathbf{Q}=0 \rightarrow \overline{\mathbf{Z}}
$$

The final equations for the $\overline{\mathbf{W}}^{\mathrm{C}}$ multiplier are given as

$$
\bar{W}_{i j \sigma}^{\mathrm{C}}=\frac{1}{2} H_{i j \sigma}[\mathbf{P}]+\sum_{\kappa \lambda} X_{\kappa i \sigma}^{\mathrm{T}}\left(\Omega S_{\kappa \lambda}-F_{\kappa \lambda \sigma}\right) X_{\lambda j \sigma}+\sum_{\kappa \lambda \sigma^{\prime}} \sum_{\eta \tau \sigma^{\prime \prime}} D_{\kappa \lambda \sigma^{\prime}}^{\mathrm{x}} D_{\eta \tau \sigma^{\prime \prime}}^{\mathrm{x}} g_{\kappa \lambda \sigma^{\prime}, \eta \tau \sigma^{\prime \prime}, i j \sigma}^{\mathrm{xC}}
$$

with the relaxed and unrelaxed difference density matrices $\mathbf{P}$ and $\mathbf{T}$ being defined as

$$
\begin{aligned}
\mathbf{P} & =\mathbf{T}+\mathbf{D}^{\mathrm{z}} \\
D_{\mu \nu \sigma}^{\mathrm{z}} & =\frac{1}{2} \sum_{k}\left(\bar{Z}_{\mu k \sigma} C_{\nu k \sigma}^{\mathrm{T}}+C_{\mu k \sigma} \bar{Z}_{\nu k \sigma}^{\mathrm{T}}\right), \\
T_{\mu \nu \sigma} & =\sum_{k} X_{\mu k \sigma} X_{\nu k \sigma}^{\mathrm{T}}-\frac{1}{2} \sum_{\kappa \lambda k l}\left(C_{\mu k \sigma} X_{\kappa k \sigma}^{\mathrm{T}} S_{\kappa \lambda} X_{\lambda l \sigma} C_{\nu l \sigma}^{\mathrm{T}}+C_{\mu l \sigma} X_{\kappa l \sigma}^{\mathrm{T}} S_{\kappa \lambda} X_{\lambda k \sigma} C_{\nu k \sigma}^{\mathrm{T}}\right) .
\end{aligned}
$$


Note that the unrelaxed difference density matrix $\mathbf{T}$ in the $\mathrm{AO}$ basis as defined in Eq. (44) corresponds to the sum of the virtual-virtual and occupied-occupied blocks of the corresponding MO matrix $\mathbf{T}^{\mathrm{MO}}$ with the mixed virtual-occupied blocks being equal to zero. Regarding the comparison to MO formulations, ${ }^{35,56}$ the matrix $\overline{\mathbf{W}}^{\mathrm{c}}$ as defined in Eqs. (37) and (41) only comprises occupied-occupied contributions while analogous MO formulations treat the combined occupied-virtual space. Contributions referring to the virtual space of Refs. ( ${ }^{35,56}$ ) are included in our formalism via $\mathbf{Q}$ projections, explicitly taken into account due to the reformulated Brillouin condition (Eq. (38)) and the $\overline{\mathbf{W}}^{\mathrm{x}}$ constraint. Furthermore, the intermediate $\mathbf{H}$ stems from the KS matrix contributions of Eq. (14) and thus the explicit formula depends on the chosen GS reference, given here for hybrid functionals with or without ADMM,

$$
\begin{aligned}
H_{i j \sigma}[\mathbf{M}] & =\sum_{\kappa \lambda \sigma^{\prime}} M_{\kappa \lambda \sigma^{\prime}}\left[2(\kappa \lambda \mid i j \sigma)-a_{\mathrm{Ex}} \delta_{\sigma \sigma^{\prime}}[(\kappa i \sigma \mid \lambda j \sigma)+(\kappa j \sigma \mid \lambda i \sigma)]+2 f_{\kappa \lambda \sigma^{\prime}, i j \sigma}^{\mathrm{XC}}\right] \\
H_{i j \sigma}^{\mathrm{ADMM}}[\mathbf{M}] & =\sum_{\kappa \lambda \sigma^{\prime}} M_{\kappa \lambda \sigma^{\prime}}\left[2(\kappa \lambda \mid i j \sigma)+2 f_{\kappa \lambda \sigma^{\prime}, i j \sigma}^{\mathrm{XC}}\right] \\
& -a_{\mathrm{Ex}} \sum_{\hat{\kappa} \hat{\lambda} \sigma^{\prime}} \delta_{\sigma \sigma^{\prime}}\left(\hat{\mathbf{U}} \mathbf{M} \hat{\mathbf{U}}^{\mathrm{T}}\right)_{\hat{\kappa} \hat{\lambda} \sigma^{\prime}}\left[\sum_{\eta \hat{\eta} \tau \hat{\tau}} C_{\eta i \sigma}^{\mathrm{T}} \hat{U}_{\hat{\eta} \eta}^{\mathrm{T}}[(\hat{\kappa} \hat{\eta} \mid \hat{\lambda} \hat{\tau})+(\hat{\kappa} \hat{\tau} \mid \hat{\lambda} \hat{\eta})] \hat{U}_{\hat{\tau} \tau} C_{\tau j \sigma}\right] \\
& -2 a_{\mathrm{Ex}}\left[\sum_{\kappa \lambda \sigma^{\prime}} \delta_{\sigma \sigma^{\prime}} M_{\kappa \lambda \sigma^{\prime}} f_{\kappa \lambda \sigma^{\prime}, i j \sigma}^{\mathrm{GGA}}+\sum_{\hat{\kappa} \hat{\lambda} \sigma^{\prime}} \delta_{\sigma \sigma^{\prime}}\left(\hat{\mathbf{U} M} \hat{\mathbf{U}}^{\mathrm{T}}\right)_{\hat{\kappa} \hat{\lambda} \sigma^{\prime}}\left[\sum_{\eta \hat{\eta} \tau \hat{\tau}} C_{\eta i \sigma}^{\mathrm{T}} \hat{U}_{\hat{\eta} \eta}^{\mathrm{T}} f_{\hat{\kappa} \hat{\lambda} \sigma^{\prime}, \hat{\eta} \hat{\tau} \sigma}^{\mathrm{GGA}} \hat{U}_{\hat{\tau} \tau} C_{\tau j \sigma}\right]\right]
\end{aligned}
$$

The linear $\overline{\mathbf{Z}}$ vector equation,

$$
\begin{aligned}
\mathbf{A} \overline{\mathbf{Z}} & =-\mathbf{R}, \\
\sum_{\kappa} \bar{Z}_{\kappa i \sigma}^{\mathrm{T}}\left[F_{\kappa \mu \sigma}-S_{\kappa \mu} \varepsilon_{i \sigma}\right]+H_{i \mu \sigma}\left[\mathbf{D}^{\mathrm{Z}}\right] & =-R_{i \mu \sigma},
\end{aligned}
$$

contains contributions on the left-hand side, which stem from the KS matrix (Eq. (13)) and are independent of the chosen excited-state kernel K (Eq. (14)). Such a dependence is solely included on the right-hand side $\mathbf{R}$, summarizing a first term stemming from the KS matrix as well as kernel contributions which have to be adjusted for ADMM and sTDA,

$$
\begin{aligned}
R_{i \mu \sigma} & =H_{i \mu \sigma}[\mathbf{T}] \\
& +2 \sum_{\kappa} X_{\kappa i \sigma}^{\mathrm{T}} K_{\kappa \mu \sigma}\left[\mathbf{D}^{\mathrm{x}}\right]-2 \sum_{\kappa k} X_{\kappa k \sigma}^{\mathrm{T}} S_{\kappa \mu} K_{i k \sigma}\left[\mathbf{D}^{\mathrm{x}}\right]+2 \sum_{\kappa \lambda \sigma^{\prime}} \sum_{\eta \tau \sigma^{\prime \prime}} D_{\kappa \lambda \sigma^{\prime}}^{\mathrm{x}} D_{\eta \tau \sigma^{\prime \prime}}^{\mathrm{x}} g_{\kappa \lambda \sigma^{\prime}, \eta \tau \sigma^{\prime \prime}, \mu i \sigma}^{\mathrm{xC}} .
\end{aligned}
$$


Finally, the gradient $L^{\zeta}$ with respect to the nuclear coordinate $\zeta$ can be written in terms of the effective difference density matrix $\Gamma$ analogously to the formulations of Refs., ${ }^{35,56}$

$$
\begin{aligned}
L^{\zeta} & =\sum_{\mu \nu \sigma}\left[h_{\mu \nu}^{\zeta}+V_{\mu \nu \sigma}^{\mathrm{xC}(\zeta)}\right] P_{\mu \nu \sigma}-\sum_{\mu \nu \sigma} S_{\mu \nu}^{\zeta} \Lambda_{\mu \nu \sigma} \\
& +\sum_{\mu \nu \kappa \lambda \sigma \sigma^{\prime}}\left[(\mu \nu \mid \kappa \lambda)^{\zeta} \Gamma_{\mu \nu \sigma \kappa \lambda \sigma^{\prime}}+f_{\mu \nu \sigma \kappa \lambda \sigma^{\prime}}^{\mathrm{xC}(\zeta)} D_{\mu \nu \sigma}^{\mathrm{x}} D_{\kappa \lambda \sigma^{\prime}}^{\mathrm{x}}\right]
\end{aligned}
$$

with the intermediates

$$
\begin{aligned}
\Lambda_{\mu \nu \sigma} & =\sum_{k l} C_{\mu k \sigma} \bar{W}_{k l \sigma}^{\mathrm{C}} C_{\nu l \sigma}^{\mathrm{T}}+\frac{1}{2} \sum_{k} \varepsilon_{k \sigma}\left(\bar{Z}_{\mu k \sigma} C_{\nu k \sigma}^{\mathrm{T}}+C_{\mu k \sigma} \bar{Z}_{\nu k \sigma}^{\mathrm{T}}\right) \\
& +\sum_{k l}\left[C_{\mu k \sigma}^{\mathrm{T}} X_{\nu l \sigma}+X_{\mu k \sigma}^{\mathrm{T}} C_{\nu l \sigma}\right] K_{k l \sigma}\left[\mathbf{D}^{\mathrm{x}}\right]+\sum_{k l}\left(\Omega+F_{k l \sigma}\right) \delta_{k l} X_{\mu k \sigma}^{\mathrm{T}} X_{\nu l \sigma}, \\
\Gamma_{\mu \nu \sigma \kappa \lambda \sigma^{\prime}} & =P_{\mu \nu \sigma} D_{\kappa \lambda \sigma^{\prime}}+D_{\mu \nu \sigma}^{\mathrm{x}} D_{\kappa \lambda \sigma^{\prime}}^{\mathrm{x}}-a_{\mathrm{Ex}} \delta_{\sigma \sigma^{\prime}}\left[P_{\mu \kappa \sigma} D_{\nu \lambda \sigma^{\prime}}+D_{\mu \kappa \sigma}^{\mathrm{x}} D_{\nu \lambda \sigma^{\prime}}^{\mathrm{x}}\right] .
\end{aligned}
$$

Note that the total nuclear forces sum contributions due to the ES energy functional and the additional constraints, as listed in Eq. (50), as well as the GS energy contributions $E_{\mathrm{GS}}^{\zeta}$.

Regarding the discussed kernel options, additional terms for the gradient have to be considered for the transformed ADMM matrix of Eq. (24), with all contributions implying the chain rule,

$$
\begin{aligned}
\frac{\partial K_{\mu \nu \sigma}^{\mathrm{EX}, \mathrm{ADMM}}\left[\mathbf{D}^{\mathrm{X}}\right]}{\partial \zeta} & \leftarrow\left(\left(\hat{\mathbf{U}}^{\mathrm{T}}\right)^{\zeta} \hat{\mathbf{K}}^{\mathrm{Ex}}\left[\hat{\mathbf{U}} \mathbf{D}^{\mathrm{x}} \hat{\mathbf{U}}^{\mathrm{T}}\right] \hat{\mathbf{U}}\right)_{\mu \nu \sigma}+\left(\hat{\mathbf{U}}^{\mathrm{T}} \hat{\mathbf{K}}^{\mathrm{Ex}}\left[\hat{\mathbf{U}} \mathbf{D}^{\mathrm{x}} \hat{\mathbf{U}}^{\mathrm{T}}\right] \hat{\mathbf{U}}^{\zeta}\right)_{\mu \nu \sigma} \\
& +\left(\hat{\mathbf{U}}^{\mathrm{T}} \hat{\mathbf{K}}^{\mathrm{Ex}}\left[\hat{\mathbf{U}}^{\zeta} \mathbf{D}^{\mathrm{x}} \hat{\mathbf{U}}^{\mathrm{T}}+\hat{\mathbf{U}} \mathbf{D}^{\mathrm{x}}\left(\hat{\mathbf{U}}^{\mathrm{T}}\right)^{\zeta}\right] \hat{\mathbf{U}}\right)_{\mu \nu \sigma} \\
& +\sum_{\hat{\mu} \hat{\nu} \hat{\kappa} \hat{\lambda}} \hat{U}_{\hat{\mu} \mu}^{\mathrm{T}}(\hat{\mu} \hat{\kappa} \mid \hat{\nu} \hat{\lambda})^{\zeta} \hat{U}_{\hat{\nu} \nu} \sum_{\kappa \lambda} \hat{U}_{\hat{\kappa} \kappa} D_{\kappa \lambda \sigma}^{\mathrm{x}} \hat{U}_{\hat{\lambda} \lambda}^{\mathrm{T}}
\end{aligned}
$$

encompassing the gradient for the ADMM projection matrix $\hat{\mathbf{U}}^{\zeta}$,

$$
\hat{\mathbf{U}}^{\zeta}=\hat{\mathbf{S}}^{-1}\left[\hat{\mathbf{V}}^{\zeta}-\hat{\mathbf{S}}^{\zeta} \hat{\mathbf{U}}\right]
$$

Analogous contributions have to be considered for the correction term of Eq. (25). sTDA kernels bear the advantage that they do not require third order derivatives of the XC kernel, but additional derivatives of the overlap matrix $\mathbf{S}^{1 / 2}, 57$

$$
\left(\frac{\partial \mathbf{S}^{1 / 2}}{\partial \zeta}\right)_{\mu \nu}=\sum_{\kappa \lambda \eta \tau} U_{\mu \kappa}^{\mathrm{S}} U_{\eta \kappa}^{\mathrm{S}}\left(\frac{\partial \mathbf{S}}{\partial \zeta}\right)_{\eta \tau} U_{\tau \lambda}^{\mathrm{S}}\left(\sqrt{s_{\lambda}}+\sqrt{s_{\kappa}}\right)^{-1} U_{\nu \lambda}^{\mathrm{S}}
$$


with the eigenvalues $\mathbf{s}$ and eigenvectors $\mathbf{U}^{\mathrm{S}}$ of the overlap matrix.

\section{Results and discussion}

\subsection{Tests for accuracy — Benchmark results for 35 main-group molecules}

To assess the accuracy of ADMM and sTDA excited-state properties, we performed a benchmark on a molecular test set by Budzak et al. containing 35 small molecules. The test set contains main-group atoms of the first and second row as well as sulfur, selenium, chlorine and bromine. Reported EOM-CCSD geometries were obtained using Gaussian16, ${ }^{58}$ correlating all electrons (including core electrons) and choosing a def2-TZVPP basis. ${ }^{59}$ The nature of the GS and ES reference structures was furthermore checked by performing frequency calculations and computing $T_{1}$ diagnostics. We selected these structures as highest-accuracy level reference and performed, for comparison, all-electron PBE0 calculations with the Turbomole program package, ${ }^{60}$ first using the def2-TZVPP basis to unravel the effect of the underlying electronic structure method and then increasing the basis set to def2-QZVPP 61 quality to get an estimate for the basis set error. Deviations of PBE0 computations with $\mathrm{CP} 2 \mathrm{~K}$ to the mentioned EOM-CCSD and PBE0 reference data originate then mainly from core shells being described in terms of pseudopotentials and correspondingly adapted basis sets. More specifically, we used pseudopotentials and basis sets which were optimized for the PBE0 functional with the numerical atom code of $\mathrm{CP} 2 \mathrm{~K}$ and which are available within the database and distribution of CP2K. ${ }^{41,62}$ To check consistency of the chosen CP2K basis sets and pseudopotentials, we investigated a hierarchy of MOLOPT as well as correlation consistent ccGRB-X basis sets. Benchmark data on vertical singlet excitation energies, geometries of the first excited state, adiabatic excitation and fluorescence energies (for PBE0, ADMMPBE0 and sTDA kernels based on PBE0 and ADMM-PBE0 references) are given for the two basis set families in comparison to both EOM-CCSD/def2-TZVPP/Gaussian, PBE0/def2TZVPP/Turbomole, and PBE0/def2-QZVPP/Turbomole references in the supplementary material, demonstrating that MOLOPT and ccGRB-X basis sets yield comparable accuracy and that converged results are in general obtained for triple-zeta basis sets. For the sake of convenience, we therefore restrict the following discussion to subsets of MOLOPT type basis sets, concentrating on the assessment of the mentioned excited-state properties regarding

a) the accuracy of ADMM in comparison to conventional hybrid functional TDDFT as well as b) the comparison of semi-empirical sTDA and ADMM-approximated hybrid functional kernels in comparison to highly accurate EOM-CCSD reference data. 
Regarding the performed benchmark, a coupling parameter with a default value of $a_{\mathrm{Ex}}=0.2$ was chosen for the exchange contribution for sTDA computations throughout the following discussion. As noted in the supplementary information (SI), adjustments only had to be made in the case of Formylchloride to avoid dissociation upon ES geometry optimization. A default value for $a_{\mathrm{Ex}}$ might not correspond to an optimal choice, but a thorough and careful optimization of $a_{\mathrm{Ex}}$ for the investigated benchmark sets and the assessed extended materials is beyond the scope of this work. The general idea of ADMM is to reduce computational costs of the exact exchange contribution by trading in basis-set incompleteness with a GGA correction term. The introduced error is then, however, dependent on both the auxiliary basis-set size as well as the chosen exchange density functional. To discriminate between the two error sources, the discussed ADMM calculations for the molecular benchmark set are performed without correction. An optimally chosen GGA exchange functional contribution could improve the discussed results, with the latter representing thus the lowest limit for the ADMM accuracy. It was e.g. shown in Ref. ${ }^{7}$ that exchange functionals like KT3X and OPTX improve over PBEX for ground-state properties, but analogous investigations for the excited state are left for future work. For the subsequently discussed assessment of computational timings for periodic systems, we however included a PBEX exchange functional correction to account for the additionally required resources.

\subsubsection{Excited-state geometries: Impact of the auxiliary basis set size for ADMM kernels}

Relaxed excited-state geometries for PBE0 and ADMM-PBE0 kernels using MOLOPT and ccGRB-X basis sets as well as sTDA kernels based on PBE0 and ADMM-PBE0 references are reported in the SI. The data includes a corresponding statistical analysis on the error in the geometrical data with respect to EOM-CCSD/def2-TZVPP, PBE0/def2-TZVPP, and PBE0/def2-QZVPP references. In contrast to the study of Ref., ${ }^{47}$ we excluded first excited states of the eclipsed structures of Nitrosomethane and Trifluoronitrosomethane as these singlet states represent transition-state structures with an imaginary frequency. Due to the large number of geometries that had to be optimized considering different kernels and basis sets, we calculated vibrational frequencies only for those structures that differed significantly from the reference geometries, ensuring that they represent local minima. In the earlier benchmarks of Ref., ${ }^{47}$ MADs for bond lengths of GS structures are predicted to be in the range of 0.5 to $1.0 \mathrm{pm}$ for hybrid functionals, with largest errors found for $\mathrm{CO}, \mathrm{CN}$, CS, and CSe bonds. In general, it is concluded that deviations are largest for polarized bonds. In Figure 1 the error introduced by ADMM in bond lengths of GS or first singlet ES geometries is depicted for selected bonds of the 35 molecules of the benchmark set, with "ADMM 
error" being defined as the deviation that results from comparing the conventional PBE0 computations with approximated ADMM-PBE0 ones choosing the same primary basis set. The assessment is thus reflecting the accuracy of the auxiliary basis set size. For both GS and ES, results relying on the smallest dzp basis, colored in green or blue depending on the chosen primary basis, deviate significantly from computations using larger auxiliary basis sets, depicted in orange and red. Predicted bond lengths are too long, with dzp errors amounting up to $-3.9 /-4.1 \mathrm{pm}$ for GS / ES geometries and being thus much larger than the corresponding error range for the tzp and tz2p auxiliary basis sets; both tzp and tz2p bonds combined with a TZVP or TZV2P primary basis deviate by at most -1.0 or $-0.9 \mathrm{pm}$. Comparing GS and ES distributions, the error spread for the ES is slightly broader than for the GS. However, both tzp distributions suggest a classification in two or more groups depending on the type of atoms involved and resulting in two maxima in the histograms. Bonds including H, S, Se and C-C bonds can be associated with relatively small errors corresponding to the first maximum and Gaussian distribution around $0.0 \mathrm{pm}$. Larger deviations in the range of -0.3 to $-0.5 \mathrm{pm}$ can be found for oxygen or halogen bonds contributing to the second maximum in both GS and ES histograms. Outliers corresponding to the maximum errors of $-1.0 \mathrm{pm}$ can be traced back to Nitrogen bonds. An analogous classification and assignment for the dzp auxiliary basis is less conclusive for the ES due to the broader distributions, but still holds true for the GS with the exception that C-C bonds fall into the error range of the second maximum around $-1.5 \mathrm{pm}$.

For comparison, the ADMM error in selected angles of the optimized ES geometries is depicted in Figure 2, demonstrating that the impact of the chosen auxiliary basis on the angles is less dependent on the basis set size than it is for the bond lengths even though the width of the Gaussian distribution is reduced when going from double to triple-zeta basis sets. An analogous plot for the GS, given in the SI, looks nearly identical with the only difference that outliers with maximum errors in the angles rising up to $18.9^{\circ}$ and $7.9^{\circ}$ are found for the $\mathrm{C}-\mathrm{C} \equiv \mathrm{C}$ and $\mathrm{C} \equiv \mathrm{C}-\mathrm{H}$ angles of the dzp structures of cyanoacetylene. For the dzp basis, the ES of this molecule is thus predicted to be significantly less bent in comparison to auxiliary basis sets with larger cardinal numbers as well as the conventional PBE0 results with a DZVP, TZVP and TZV2P primary basis. Outliers were checked to confirm they are local minima, but further characterization of the excited state would be needed to draw final conclusions. However, smaller angles, as predicted by the dzp auxiliary basis, are in better agreement with EOM-CCSD and experimental reference data ${ }^{63}$ - an agreement which might be due to fortuitous error cancellation.

A similar and transferable result is found for dihedral angles investigated for a subset of 13 molecules for which excitation leads to a significantly bent excited-state structure. While 
Figure 1: Error in [pm] for selected bond lengths for optimized ground- and first singlet excited-state geometries comparing conventional PBE0 and ADMM-PBE0 computations for different auxiliary $(\mathrm{ABS})$ and primary basis sets (PBS) indicated as ABS / PBS.
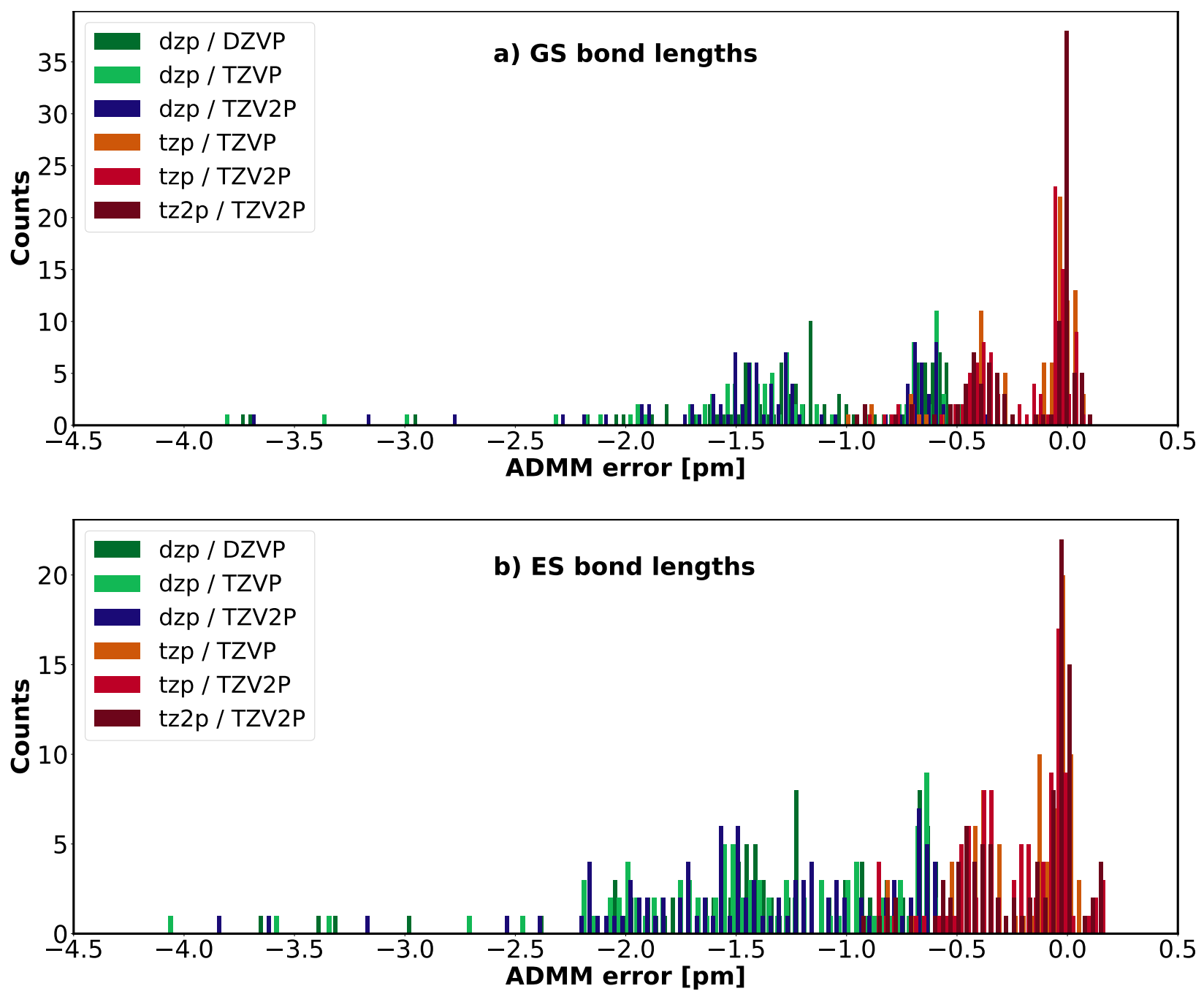
Figure 2: Error in $\left[^{\circ}\right.$ for selected angles for optimized first singlet excited-state geometries comparing conventional PBE0 and ADMM-PBE0 computations.

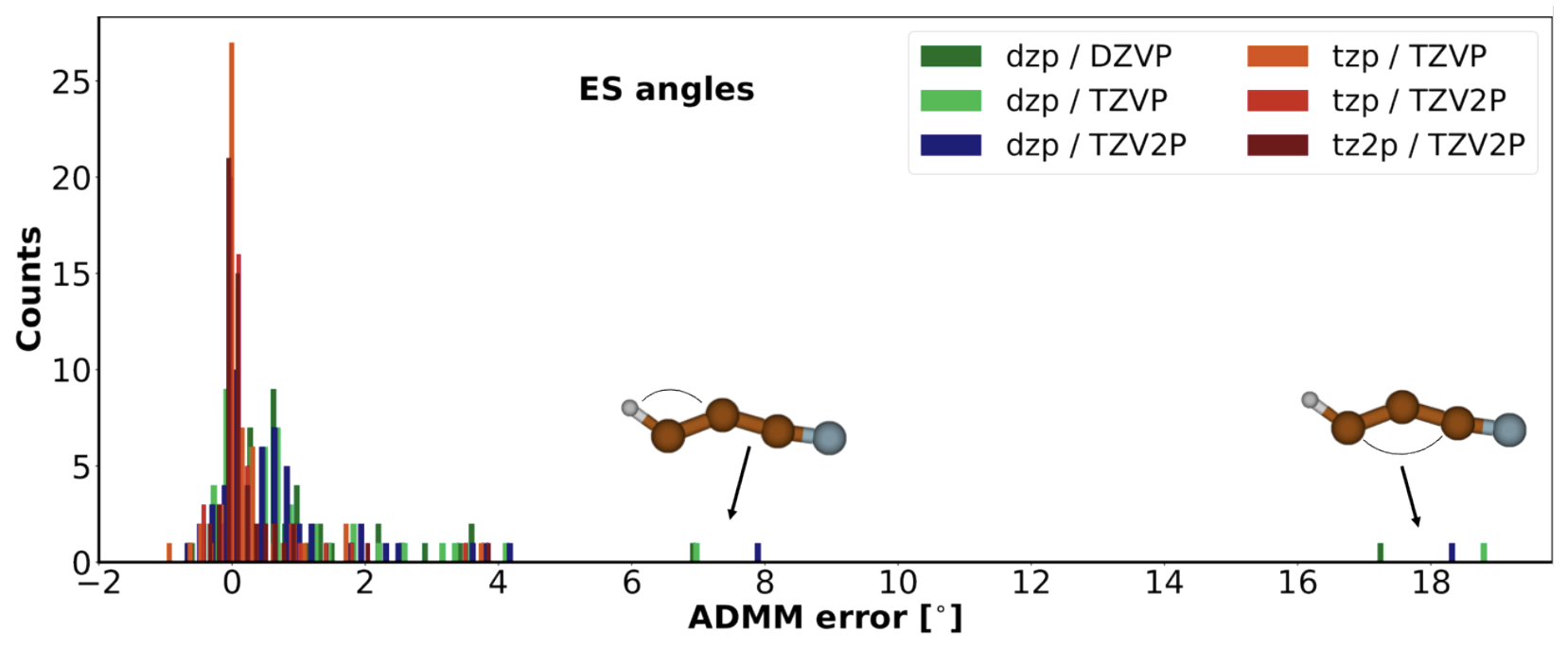

dihedral angles of corresponding GS geometries are consistently predicted to describe a planar geometry for all auxiliary and primary basis set sizes, the bending angle of the excited state is more accurately described when increasing the cardinal number of the auxiliary basis from double- to triple-zeta size. Again the effect of additional polarization functions as included within the largest tz2p basis is negligible. A plot for the ADMM error in dihedral angles analogous to Figures 1 and 2 is given in the SI.

Taking a summarizing look at the corresponding statistical data of Table 1, listing mean deviations (MDs), mean absolute deviations (MADs), and standard deviations (STDs) for bond lengths, angles, and dihedral angles of ES geometries optimized within the various primary and auxiliary basis set combinations, emphasizes again the impact of the chosen auxiliary basis: while MADs of the dzp basis amount up to $1.4 \mathrm{pm}$ for bond lengths and up to $1.2^{\circ}$ or $2.1^{\circ}$ for bond and dihedral angles, tzp results are converged with remaining deviations of $0.2 \mathrm{pm} / 0.3^{\circ} / 0.3^{\circ}$. The deviations are negligibly small in comparison to the error of 0.5 to $1.0 \mathrm{pm}$ that was found for bond lengths for hybrid functionals in comparison to highly accurate coupled-cluster results. ${ }^{64}$ Error distributions are relatively broad for the dzp auxiliary basis and nearly equally narrow for tzp and tz2p basis sets. MDs and MADs are of the same magnitude, indicating that the dzp auxiliary basis predicts bonds that are consistently too long. This trend is in agreement with the conclusions of Ref., ${ }^{47}$ with the latter stating that global and range-separated hybrids yield slightly too short bonds and that functionals with a rather small amount of exact exchange show negative mean signed errors. 
Table 1: Statistics including mean deviations (MDs), mean absolute deviations (MADs), and standard deviations (STDs) for different auxiliary and primary basis sets (ABS/PBS) visualizing that the ADMM error in ES bond lengths [pm], angles [ ${ }^{\circ}$, and dihedral angles $\left[^{\circ}\right.$ ] as well as in the first adiabatic excitation $\left(E_{\text {ad }}\right)$, the first 10 vertical excitation $\left(E_{\text {vert }}\right)$, and the first fluorescence $\left(E_{\mathrm{fl}}\right)$ energy $[\mathrm{eV}]$ is converged for auxiliary basis sets of triple-zeta size.

\begin{tabular}{|c|c|c|c|c|c|c|c|c|c|}
\hline \multirow[t]{2}{*}{ ABS/PBS } & \multicolumn{3}{|c|}{ Bonds [pm] } & \multicolumn{3}{|c|}{ Angles [ $\left.{ }^{\circ}\right]$} & \multicolumn{3}{|c|}{ Dihedrals [ } \\
\hline & MD & MAD & STD & MD & MAD & STD & MD & MAD & STD \\
\hline dzp/DZVP & -1.33 & 1.33 & 0.62 & 1.11 & 1.21 & 2.54 & -2.14 & 2.14 & 1.17 \\
\hline dzp/TZVP & 39 & 1.39 & 0.66 & 1.11 & 1.23 & 2.74 & -2.00 & 2.00 & 1.10 \\
\hline $\mathrm{dzp} / \mathrm{TZV} 2 \mathrm{P}$ & 39 & 1.39 & 0.64 & 1.13 & 1.23 & 2.72 & -2.11 & 2.11 & 1.18 \\
\hline tzp/TZVP & -0.21 & 0.23 & 0.25 & 0.20 & 0.33 & 0.66 & -0.11 & 0.17 & 0.18 \\
\hline tzp/TZV2P & -0.21 & 0.22 & 0.23 & 0.22 & 0.29 & 0.60 & -0.27 & 0.29 & 0.20 \\
\hline tz2p/TZV2P & -0.21 & 0.23 & 0.26 & 0.20 & 0.28 & 0.62 & .16 & 0.17 & 0.12 \\
\hline \multirow[t]{2}{*}{$\mathrm{ABS} / \mathrm{PBS}$} & \multicolumn{3}{|c|}{$E_{\text {ad }}[\mathrm{eV}]$} & \multicolumn{3}{|c|}{$E_{\text {vert }}[\mathrm{eV}]$} & \multicolumn{3}{|c|}{$E_{\mathrm{fl}}[\mathrm{eV}]$} \\
\hline & MD & MAD & STD & $\overline{M D}$ & MAD & STD & $\mathrm{MD}$ & MAD & STD \\
\hline $\mathrm{dzp} / \mathrm{DZVP}$ & 02 & 0.044 & 0.061 & 030 & 91 & 0.117 & 0.043 & .084 & 0.094 \\
\hline /TZVP & 008 & 0.049 & 0.063 & 0.035 & 0.089 & 0.111 & 0.045 & 0.087 & 0.097 \\
\hline$/ \mathrm{TZV} 2 \mathrm{P}$ & 009 & 0.048 & 0.061 & 0.036 & 0.088 & 0.108 & 0.048 & 0.085 & 0.092 \\
\hline /TZVP & .007 & 0.020 & 0.030 & 0.005 & 0.035 & 0.050 & -0.003 & 0.025 & 0.037 \\
\hline tzp/TZV2P & 004 & 0.019 & 0.029 & 0.009 & 0.036 & 0.049 & -0.001 & 0.024 & 0.036 \\
\hline tz2p/TZV2P & -0.006 & 0.018 & 0.028 & 0.014 & 0.033 & 0.046 & -0.001 & 0.022 & 0.022 \\
\hline
\end{tabular}

\subsubsection{Excited-state geometries: ADMM and sTDA kernels in comparison to EOM-CCSD references}

To analyze the performance of ADMM-PBE0 and sTDA kernels in comparison to highly accurate EOM-CCSD reference data, we restricted investigations to basis sets of triple-zeta size. In Figure 3 the error in the geometrical data is given as normal distributions based on the corresponding MDs and STDs; more detailed statistical analysis and explicit errors listed for each molecule can be found in the SI. To give not only an upper limit for a desired high-accuracy reference, but also a lower reference point of reachable performance, conventional hybrid functional PBE0 results are depicted in black, deviating by MDs of 0.18 $\mathrm{pm} /-0.75^{\circ} /-1.18^{\circ}$ from the EOM-CCSD reference. As for the preceding assessment on the ADMM auxiliary basis size, PBE0 results represent the basis set limit for ADMM computations, a fact which is reflected in the similarity of the PBE0 error distributions with the ones obtained for analogous ADMM computations, colored in orange and red for a tzp auxiliary and a TZVP or TZV2P primary basis, respectively. In comparison, error distributions for sTDA kernels are increasingly broadened when going from PBE0/TZVP to ADMM-PBE0/TZVP + tzp references. The deviation of the semi-empirical results is most pronounced for dihedral angles, with maximum errors amounting up to $-13.29^{\circ}$ and $-14.1^{\circ}$ for $\mathrm{PBE} 0 / \mathrm{TZVP}$ and ADMM-PBE0/TZVP+tzp references. 
Figure 3: Normal distribution based on the mean deviation (MD) and standard deviation (STD) wrt EOM-CCSD reference geometries in $[\mathrm{pm}] /\left[^{\circ}\right] /\left[^{\circ}\right.$ for selected bond lengths / angles / dihedral angles for optimized first singlet excited-state geometries comparing conventional PBE0, ADMM-PBE0 and sTDA computations using triple-zeta basis sets.
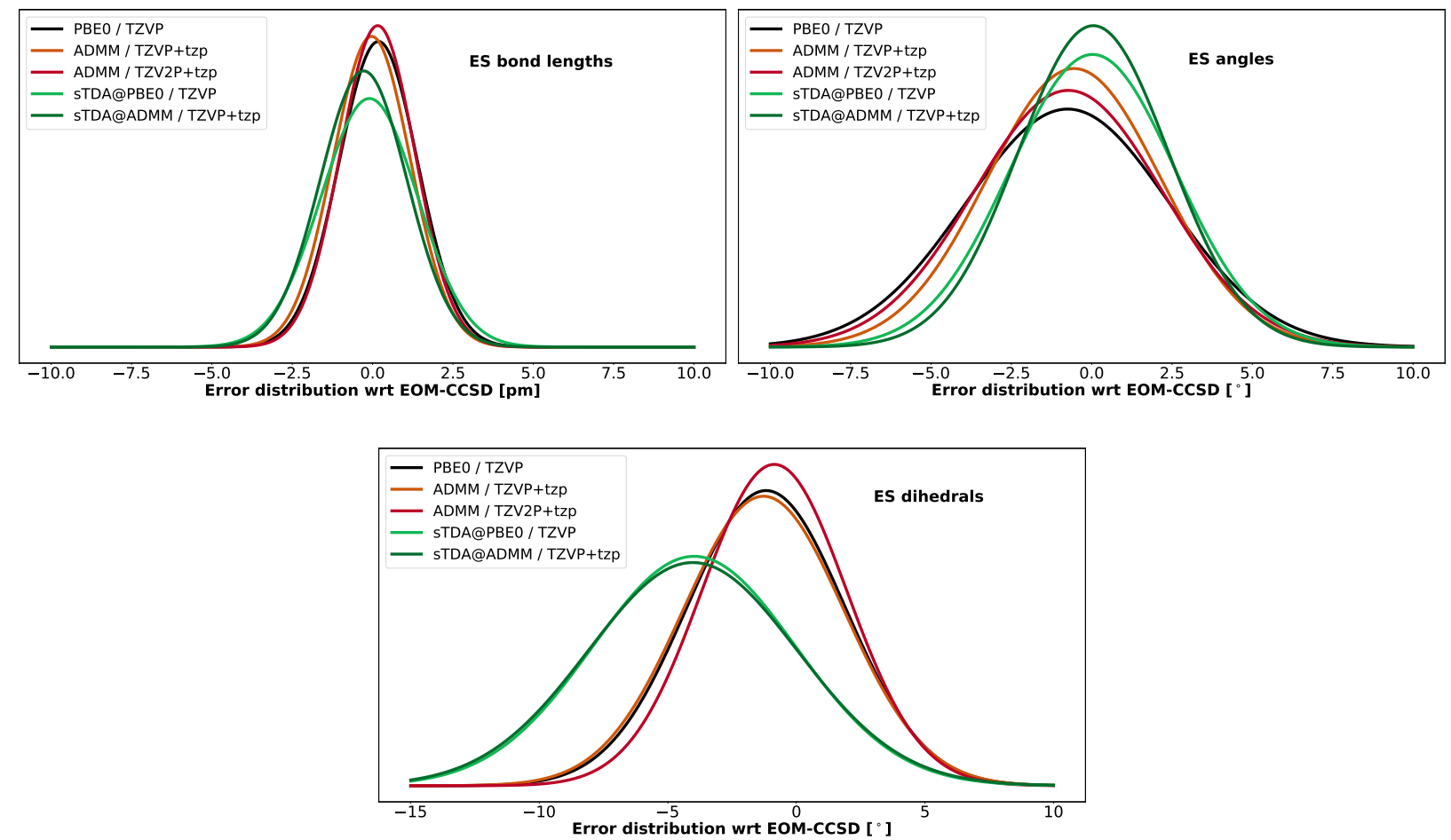

Table 2: Statistics including mean deviations (MDs), mean absolute deviations (MADs) and standard deviations (STDs) visualizing the error for both ADMM-PBE0 and sTDA kernels for excited-state geometries comprising ES bond lengths [pm], angles $\left[{ }^{\circ}\right]$ and dihedral angles $\left[{ }^{\circ}\right]$ as well as first adiabatic excitation $\left(E_{\text {ad }}\right)$, vertical excitation $\left(E_{\text {vert }}\right)$ and fluorescence energies $\left(E_{\mathrm{ff}}\right)[\mathrm{eV}]$. Errors in the geometrical data and $E_{\mathrm{ad}}$ are calculated with respect to the EOM-CCSD reference data of Ref.; ${ }^{47}$ errors in $E_{\text {vert }}$ and $E_{\mathrm{fl}}$ are referring to PBE0/def2QZVPP/TURBOMOLE reference computations.

\begin{tabular}{|ll|ccc|ccc|ccc|}
\hline \multirow{2}{*}{ Kernel } & \multirow{2}{*}{ PBS+ABS } & \multicolumn{3}{|c|}{ Bonds [pm] } & \multicolumn{3}{c|}{ Angles [ $\left.{ }^{\circ}\right]$} & \multicolumn{3}{c|}{ Dihedrals $\left[^{\circ}\right]$} \\
\cline { 3 - 11 } & & MD & MAD & STD & MD & MAD & STD & MD & MAD & STD \\
\hline PBE0 & TZVP & 0.18 & 0.96 & 1.22 & -0.75 & 1.59 & 3.16 & -1.18 & 2.19 & 3.07 \\
ADMM-PBE0 & TZVP+tzp & -0.03 & 0.94 & 1.20 & -0.55 & 1.42 & 2.70 & -1.28 & 2.23 & 3.13 \\
sTDA@PBE0 & TZVP & -0.10 & 1.06 & 1.50 & 0.03 & 1.60 & 2.57 & -3.98 & 4.06 & 3.95 \\
sTDA@ADMM-PBE0 & TZVP+tzp & -0.28 & 1.03 & 1.35 & 0.05 & 1.51 & 2.34 & -4.03 & 4.14 & 4.06 \\
\hline & & \multicolumn{3}{|c|}{$E_{\text {ad }}[\mathrm{eV}]$} & & \multicolumn{3}{c|}{$E_{\text {vert }}[\mathrm{eV}]$} & & \multicolumn{3}{c|}{$E_{\mathrm{ff}}[\mathrm{eV}]$} \\
\cline { 3 - 11 } & & MD & MAD & STD & MD & MAD & STD & MD & MAD & STD \\
\hline PBE0 & DZVP & 0.27 & 0.37 & 0.42 & 0.16 & 0.16 & 0.12 & 0.16 & 0.16 & 0.10 \\
PBE0 & TZVP & 0.26 & 0.36 & 0.41 & 0.15 & 0.15 & 0.11 & 0.15 & 0.15 & 0.10 \\
PBE0 & TZV2P & 0.24 & 0.34 & 0.41 & 0.13 & 0.13 & 0.12 & 0.12 & 0.13 & 0.10 \\
ADMM-PBE0 & TZVP+tzp & 0.25 & 0.36 & 0.41 & 0.13 & 0.13 & 0.10 & 0.15 & 0.15 & 0.08 \\
sTDA@PBE0 & TZVP & -0.23 & 0.34 & 0.39 & -0.45 & 0.45 & 0.18 & -0.27 & 0.30 & 0.21 \\
sTDA@ADMM-PBE00 & TZVP+tzp & -0.10 & 0.33 & 0.44 & -0.34 & 0.34 & 0.17 & -0.14 & 0.18 & 0.16 \\
\hline
\end{tabular}




\subsubsection{Vertical excitation energies}

Benchmarking excited-state methods with the focus on vertical excitation energies has been established as a common assessment tool (see e.g. Ref. ${ }^{65}$ and references therein). Here one assumes that excitations occur without a change in geometry. Corresponding studies showed that TDDFT excitation energies, which are classified as Rydberg states or associated with a significant amount of charge transfer, are underestimated leading to errors in the order of several eV. ${ }^{66} \mathrm{~A}$ common remedy is to include exact exchange, suggesting that the investigated ADMM-approximated hybrid kernel as well as the sTDA kernel with its motivation to capture the correct physics and asymptotics of electronic interactions could be well-suited compromises to retain sufficient accuracy while reaching high efficiency for a broad range of applications. Analyzing the nature of a transition is beyond the scope of the current work and, for the sake of convenience, we restricted the analysis of vertical excitation energies to a direct comparison of the first 10 excitation energies with states being assigned solely by the corresponding ES energy. Such a simplified comparison might not be justified and lead to wrong assignments when comparing different basis set sizes, but should give a valid assessment of the accuracy of the different kernels for basis sets of the same type. Figure 4 is displaying normal distributions based on the MD and STD for the first 10 vertical excitation energies for optimized geometries, comparing ADMM-PBE0 computations with conventional PBE0 results as well as the performance of PBE0, ADMM-PBE0 and sTDA kernels with respect to a PBE0/TZV2P/CP2K and a PBE0/def2-QZVPP/TURBOMOLE reference. Corresponding statistical data is furthermore summarized in Tables 1 and 2 as well as in the SI. As shown by the upper plot on the left, the auxiliary basis set size reduces - in analogy to our findings for the geometrical data - the ADMM error in the vertical excitation energies when increasing from double-zeta to triple-zeta size and brings only little further improvement when adding polarization functions. Taking the PBE0 computations within the largest TZV2P basis as reference exposes the impact of the increasingly large primary basis set as visualized in the upper plot on the right: PBE0 / DZVP as well as ADMM-PBE0 / DZVP + dzp computations show an equally broad error distribution with MDs and STDs rising up to $-0.39 \mathrm{eV}$ and $0.71 \mathrm{eV}$, respectively. On the other end of the accuracy spectrum, ADMM-PBE0 / TZV2P +tz2p results have a relatively sharp distribution with a ME and STD of $0.01 \mathrm{eV}$ and $0.22 \mathrm{eV}$. TZVP errors for the PBE0, ADMM-PBE0 and sTDA kernels lie in between those extreme values, highlighting that ADMM has only a negligible impact on excitation energies and that MDs of sTDA are below 0.44 or $0.36 \mathrm{eV}$ depending on the chosen GS reference. This result is in agreement with the error range of $0.2-0.5 \mathrm{eV}$ of the original molecular benchmark studies. ${ }^{21,22}$ 
Figure 4: Normal distribution based on the mean deviation (MD) and standard deviation (STD) for vertical excitation energies in $[\mathrm{eV}]$ depicting the error introduced by ADMM-PBE0 in comparison to conventional PBE0 computations (upper left plot), the performance of PBE0, ADMM-PBE0 and sTDA kernel computations using double- and triple-zeta basis sets in comparison to a PBE0/TZV2P/CP2K reference (upper right plot) as well as an analogous assessment of the kernels with respect to a PBE0/def2-QZVPP/Turbomole reference (lower plot).
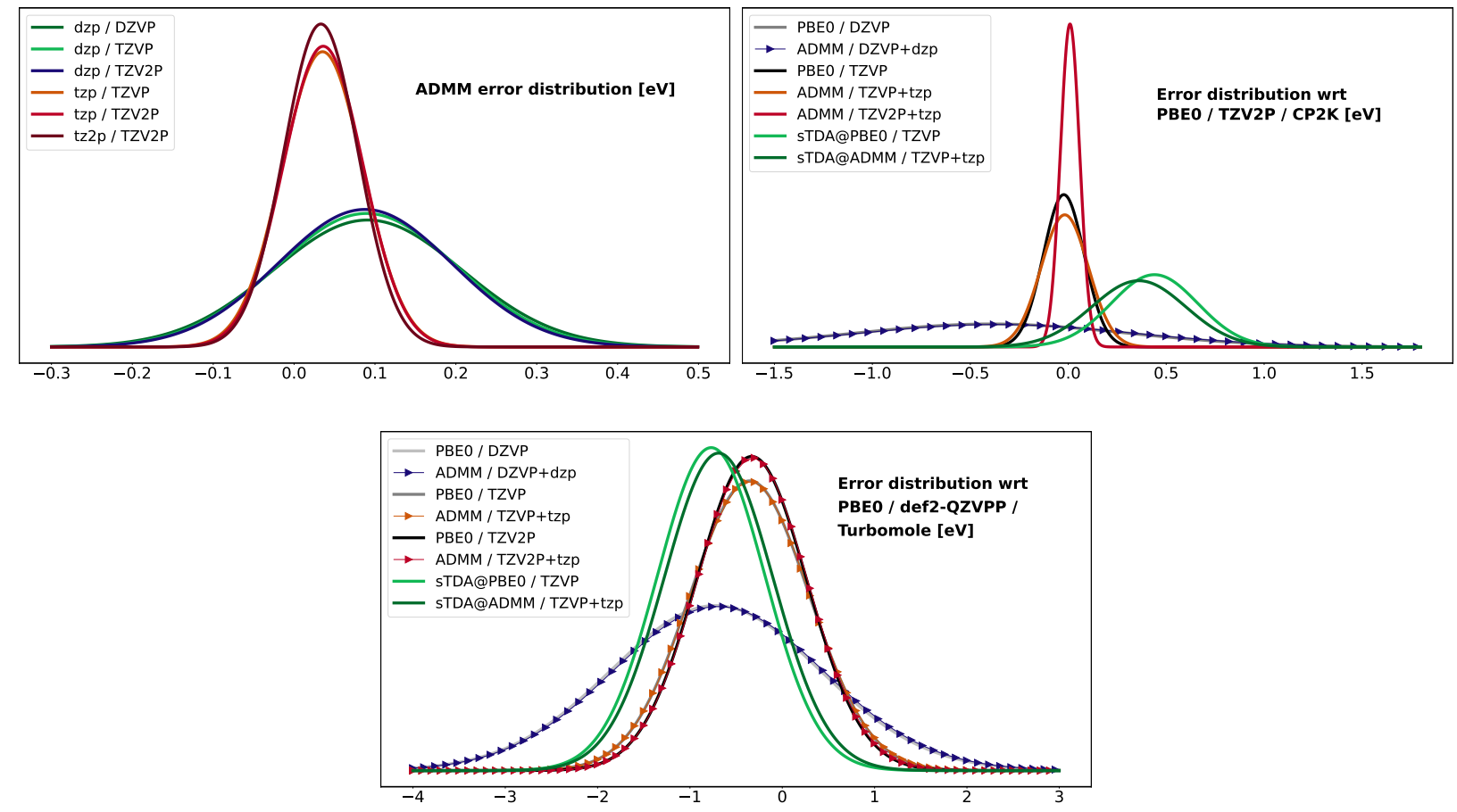


\subsubsection{Adiabatic excitation energies and fluorescence}

Adiabatic excitation energies, defined as the energy difference between lowest vibrational levels of the relaxed ground- and excited-state energies, are often benchmarked to assess ES methods. In contrast to vertical excitation energies, adiabatic excitations have the advantage of being experimentally observable and less structure sensitive. They show not a linear, but a quadratic dependence on nuclear displacements. ${ }^{67}$ The difference between calculated vertical and adiabatic excitation energy is the ES relaxation energy and fluorescence energies are defined as vertical de-excitation energies. The error in adiabatic excitation and fluorescence energies for the investigated 35 molecules is depicted in Figures 5 and 6, each figure showing in its upper plot ADMM errors for different auxiliary basis set sizes and in the lower plot the performance of ADMM and sTDA kernels. The latter is assessed for adiabatic excitation energies with respect to EOM-CCSD reference data and for fluorescence energies with respect to the performed PBE0/def2-QZVPP/Turbomole computations. Despite the varying references, ADMM errors are comparable for both excitation and de-excitation process, ranging from -0.2 to 0.2 or $0.3 \mathrm{eV}$ for the smallest dzp auxiliary basis. tzp and tz2p auxiliary basis sets reduce the maximum error to 0.1 or $0.12 \mathrm{eV}$, respectively. Corresponding MDs are of meV magnitude and MADs smaller than $0.03 \mathrm{eV}$. Analysing the different kernels in the lower plots highlights that sTDA errors are consistently shifted to either smaller or more negative errors. The correlation between the relative shift of sTDA errors with respect to both PBE0 and EOM-CCSD results and the considered amount of exact exchange was not investigated any further, but will be part of future work. However, even when choosing a non-optimized fraction of 0.2 for the coupling parameter $a_{\mathrm{Ex}}$ of Eq. 29, sTDA curves are close to the hybrid functional results achieving statistical measures of the same order of magnitude, with MDs of $0.2 / 0.3 / 0.3 \mathrm{eV}$, MADs of $0.3 / 0.4 / 0.4 \mathrm{eV}$ and maximum errors of $1.3 / 1.3 / 1.4 \mathrm{eV}$ for sTDA / ADMM-PBE0 / PBE0 adiabatic excitation energies and MDs of $-0.3 / 0.2$ / $0.2 \mathrm{eV}, \mathrm{MADs}$ of $0.3 / 0.2 / 0.2 \mathrm{eV}$ and maximum errors of $-0.4 / 0.4 / 0.6 \mathrm{eV}$ for sTDA / ADMM-PBE0 / PBE0 fluorescence energies.

\subsection{Increasing computational efficiency - Treating extended sys- tems using ADMM-PBE0 and sTDA kernels}

To analyze computational timings of ADMM-PBE0 and sTDA excited-state properties and to demonstrate the suitability of the two approaches for large-scale periodic systems, we investigated a series of porous covalent organic framework (COF) materials taken from the CURATED COFs database. ${ }^{48,49}$ The chosen subset includes the prototypes of the first ever synthesized $\mathrm{COFs}^{68}$ as well as pairs of COFs which were demonstrated to show more or less 
Figure 5: Error in adiabatic excitation energies [in eV] comparing a) approximated ADMMPBE0 and conventional PBE0 results for different auxiliary basis set sizes and b) analysing the error of PBE0, ADMM-PBE0, and sTDA kernels with respect to EOM-CCSD reference data.
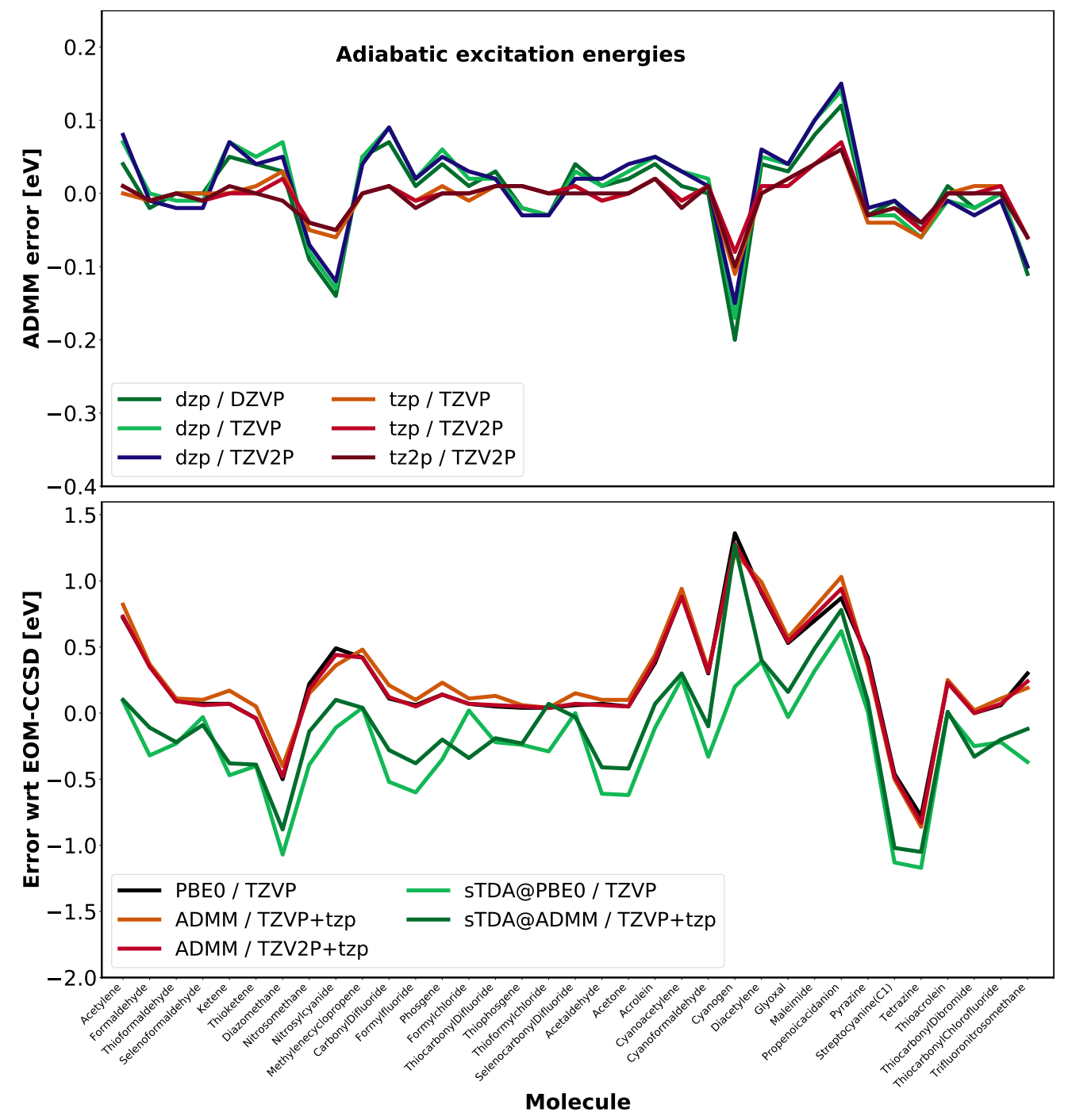
Figure 6: Error in the fluorescence energy from the first excited singlet state [in eV] comparing a) approximated ADMM-PBE0 and conventional PBE0 results for different auxiliary basis set sizes and b) analysing the error of PBE0, ADMM-PBE0, and sTDA kernels with respect to PBE0/def2-QZVPP/Turbomole reference data.
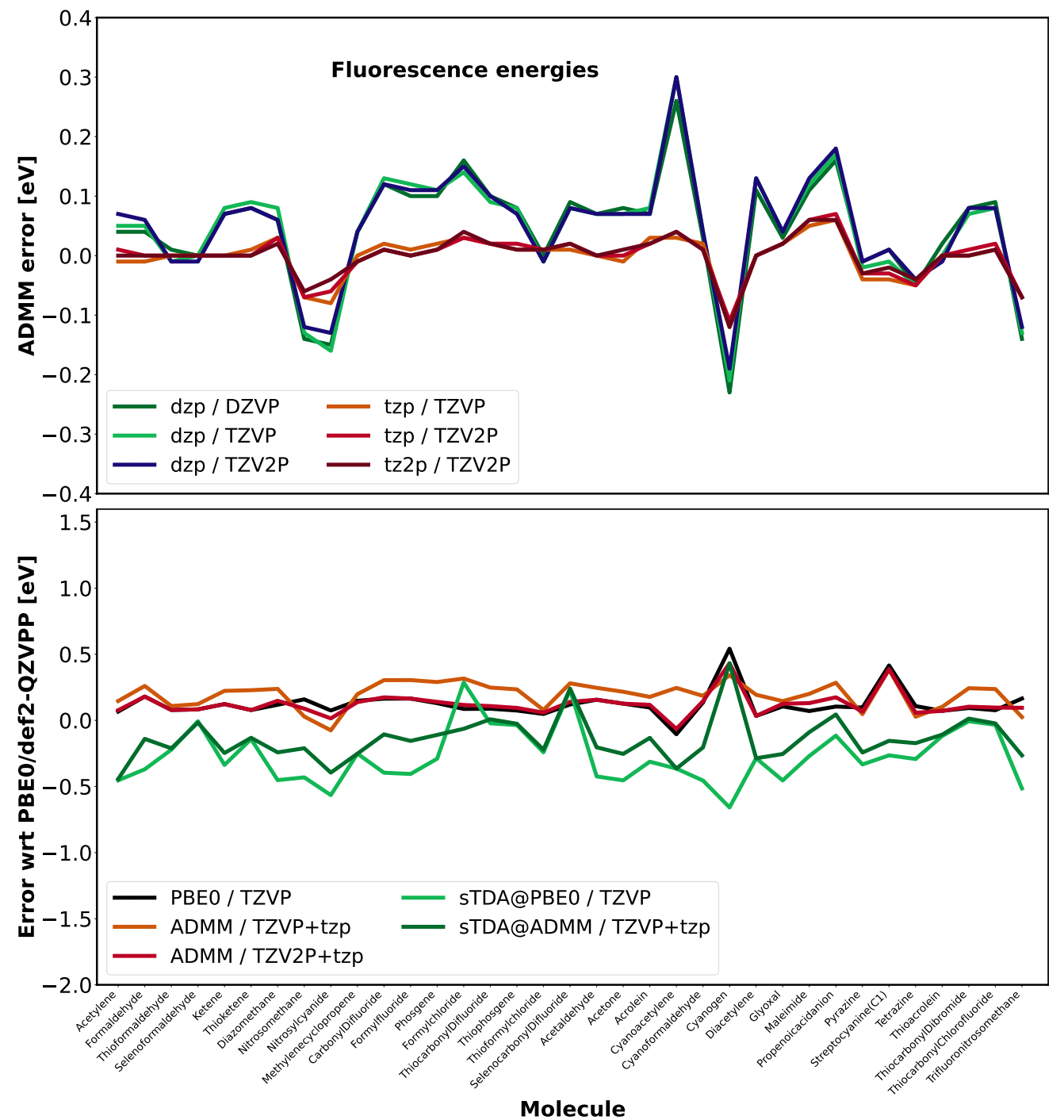
bright fluorescence depending on either accordingly tuned linker molecules or the surrounding solvent. ${ }^{69,70}$ The CURATED COFs database provides cleaned-up DFT-optimized GS structures at GGA level of accuracy, obtained with PBE functional, D3(BJ) correction, and DZVP-MOLOPT-SR basis sets. We re-optimized these geometries using a corresponding ADMM-PBE0 GS reference and triple-zeta ccGRB-T primary and tzp auxiliary basis sets to ensure a consistent accuracy of both structural and electronic GS and ES properties. The re-optimized ADMM-PBE0 GS and ES geometries are provided as supplementary information. In all calculations a truncated Coulomb operator with a radius of $4 \AA$ has been used. In Table 3, detailed timings for the first $3 \mathrm{ES}$ geometry optimization steps are given for COF 05000N2 for ADMM-approximated and sTDA kernels in comparison to conventional PBE0 results with the latter relying on an exact analytical evaluation of the two-electron-four-center exchange integrals. The total computation time of $\approx 100$ hours for a ccGRB-T basis with 2076 basis functions reflects that an analytical computation of exchange integrals within a large primary orbital basis cannot provide optimal efficiency for large periodic systems. The fact that $28 \%$ and $71 \%$ of the PBE0 computation time are solely attributed to the computation of electron repulsion integrals (ERIs) and derivative electron repulsion integrals (DERIs) emphasizes the need for approximations. Applying ADMM with a triple-zeta auxiliary basis and thus reducing the basis-set size by a factor of 2 to 1164 functions, accelerates the computation by a factor of $\approx 20$ with a remaining total time of 3.8 minutes. The small auxiliary basis reduces the number of ERIs and, in combination with increased sparsity and thus more efficient Schwarz screening, the reduction of the basis size results in timings for the integral evaluation that are reduced by a factor of $\approx 10$. More drastic is even the speed-up for the computation of DERIs due to the here applied density-matrix screening. In comparison to PBE0, the computation of DERIs is 300 times faster for ADMM-PBE0 and thus represents not $71 \%$, but solely $6 \%$ of the total costs. Timings comparable to ADMM-PBE0 can also be achieved with the semi-empirical tight binding setup: sTDA reduces the total costs to 2.4 minutes, thus by a factor of $\approx 40$ in comparison to conventional PBE0 and by a factor of $\approx 2$ in comparison to ADMM. The speed-up with respect to ADMM is due to the faster ERI evaluation relying on the semi-empirical description of both Coulomb and exchange contributions when solving the ES eigenvalue problem. sTDA and ADMM timings are in the same order of magnitude, but further acceleration could be expected when switching to a complete semi-empirical setup for both GS and ES. We are also aware that absolute timings for the sTDA ES gradient computations could still be improved by further code optimization. To give an estimate on the required relative costs of ADMM and sTDA for the computation of broad-band absorption spectra and for the geometry optimization of low-lying excited states we report timings for all six COFs in Table 4. Computations were performed for the lowest 
Table 3: Explicit computational timings [s] for COF 05000N2 comprising 84 atoms / 276 electrons in the unit cell for PBE0, ADMM-PBE0 and sTDA@ADMM-PBE0 kernels. Computations were performed using a ccGRB-T primary basis (2076 basis functions) and a tzp auxiliary basis (1164 basis functions). Timings are reported for a AMD EPYC 7742 processor using in total 1024 cores (256 MPI tasks with 4 OpenMP threads each), analysing the cost for converging the GS self-consistent field (SCF) computation, for calculating the ES energy and gradient as well as for the computation of the electron repulsion integrals (ERI) needed for the ES energy calculation and the derivative integrals (DERI) needed for the ES gradient.

\begin{tabular}{|l|ccc|}
\hline & PBE0 & ADMM-PBE0 & sTDA@ADMM-PBE0 \\
\hline GS SCF & 835 & 67 & 70 \\
ES energy and gradient & 5151 & 163 & 72 \\
ERI for ES energy & 1653 & 131 & 72 \\
DERI for ES gradient & 4276 & 13 & 10 \\
\hline Total computation time & 5986 & 230 & 142 \\
\hline
\end{tabular}

500 excited states and the geometry optimization of the first ES when selecting a manifold of 8 singlet states. The investigated system sizes range from unit cells of 84 to 300 atoms and include basis set sizes of 2076 to 7440 basis functions, respectively. Comparing the computational timings for the calculation of the lowest 500 excitation energies clearly shows the advantage of the semi-empirical setup. While computations using the ADMM kernel take $\approx 2$ to 8.5 hours, sTDA is one order of magnitude faster with a maximum computation time of less than 24 minutes. With the current setup, the tight binding approach is however less favorable for ES geometry optimization. First, the number of required optimization cycles is increased for the two COFs 05000N2 and 05001N2. Second, the averaged computation time per optimization cycle is accelerated only by a factor of 2.5 to 4 , so that ADMM-PBE0 and sTDA computation times are of the same order of magnitude and for the two COFs 05000N2 and 05001N2, the total sTDA timings even exceed the corresponding ADMM ones. For both ADMM-PBE0 and sTDA computations, timings of 5-30 minutes per optimization cycle and total timings of 1.5 - 12 hours for an ES geometry optimization can thus be expected for periodic systems in the size range of hundreds of atoms and using the indicated computational resources. 
Table 4: Computational timings [s] for ADMM-PBE0 and sTDA@ADMM-PBE0 kernels for a series of fluorescent COFs as required for the computation of broad-band absorption spectra over the lowest 500 excited states as well as for the ES geometry optimization of the first excited state when selecting a manifold of the lowest 8 singlet states. Timings are reported for a AMD EPYC 7742 processor using in total 1024 cores (256 MPI tasks with 4 OpenMP threads each).

\begin{tabular}{|l|r|r|r|r|r|r|r|r|}
\hline & \# Atoms & \multirow{2}{*}{ \# Basis } & \multicolumn{1}{|c|}{ Absorption Spectra } & \multicolumn{3}{|c|}{ 1. ES optimization } \\
\cline { 4 - 8 } & & & ADMM & sTDA & \multicolumn{2}{|c|}{ ADMM } & \multicolumn{2}{|c|}{ sTDA } \\
\cline { 4 - 8 } & & & Time & Time & Time & \# Steps & Time & \# Steps \\
\hline 05000N2 & 84 & 2076 & 6908 & 522 & 5608 & 18 & 6130 & 50 \\
05001N2 & 192 & 4848 & 23258 & 1447 & 20142 & 11 & 25036 & 55 \\
15100N2 & 300 & 7440 & 30995 & 2515 & 23473 & 22 & 11316 & 27 \\
15101N2 & 192 & 5028 & 22636 & 1328 & 44242 & 50 & 12273 & 47 \\
20610N2 & 240 & 5700 & 21145 & 1639 & 34452 & 51 & 9830 & 54 \\
20611N2 & 262 & 6188 & 23157 & 1884 & 58916 & 63 & 12508 & 50 \\
\hline
\end{tabular}

To compare the relative accuracy of ADMM-PBE0 and sTDA for periodic systems, the computed broad-band spectra are depicted in Figure 7. Corresponding vertical excitation, adiabatic excitation, and fluorescence energies, as well as Stoke's shifts are listed in Table 5. As highlighted by the six plots of Figure 7, sTDA and ADMM provide absorption spectra with qualitatively matching oscillator strengths. sTDA excitations are, however, in all cases shifted to smaller energies, with MADs for the first and the first ten vertical excitation energies amounting both up to $0.35 \mathrm{eV}$. Since the exchange mixing parameter $a_{\mathrm{Ex}}$ was set to the same value as for the molecular systems $\left(a_{\mathrm{Ex}}=0.2\right)$, it remains to be investigated if this apparently systematic shift could be improved by adjusting the amount of exchange. However, even with this default setting, the MAD in adiabatic excitation and fluorescence energies is within the error range found for molecular systems. ADMM-PBE0 and sTDA energies differ by 0.42 or $0.31 \mathrm{eV}$, respectively. Corresponding Stoke's shifts are thus also predicted with a relatively small deviation of $0.13 \mathrm{eV}$. 
Figure 7: Comparison of ADMM-PBE0 and sTDA absorption spectra for a series of fluorescent COFs.

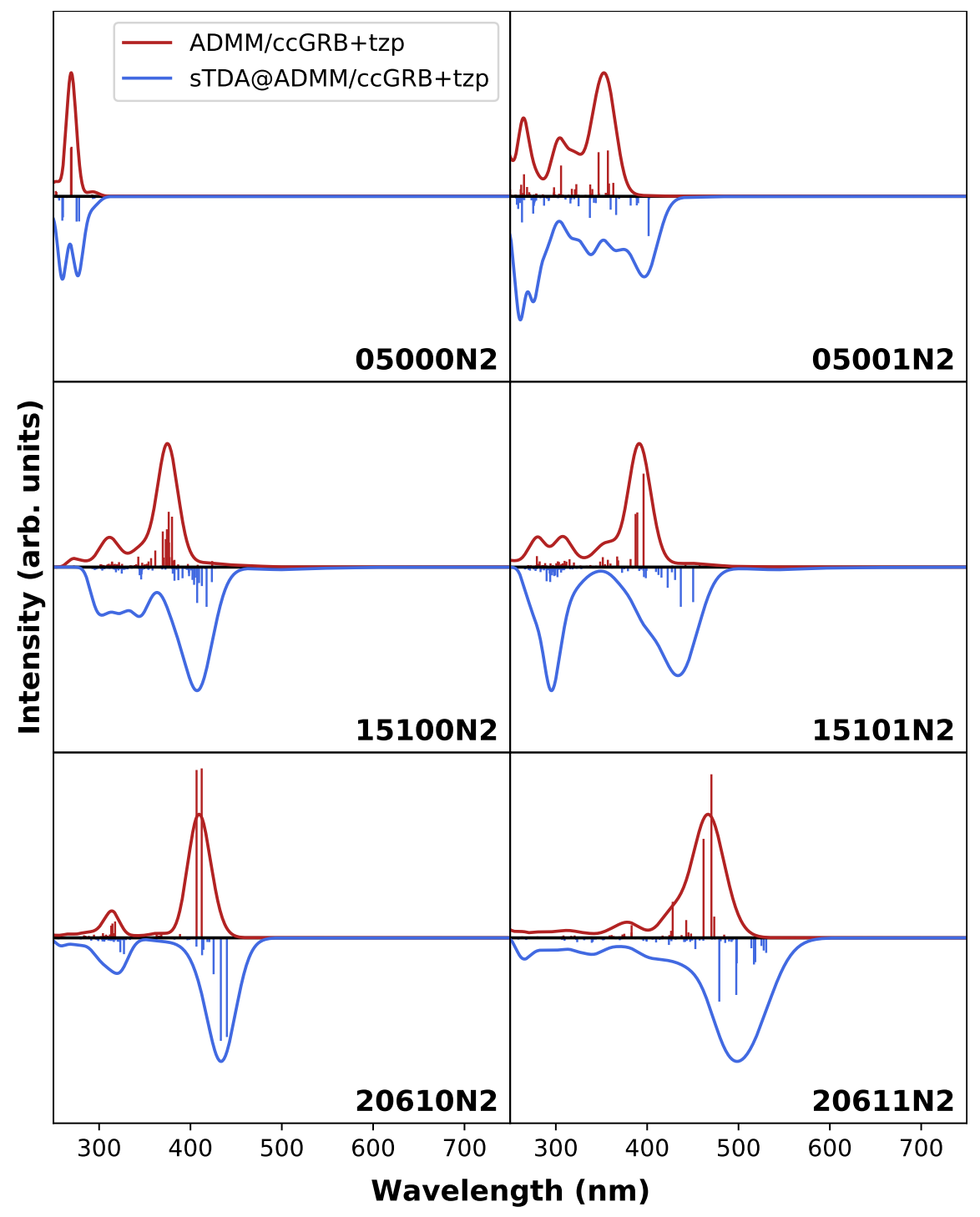


Table 5: Vertical excitation $\left(E_{\text {vert }}\right)$, adiabatic excitation $\left(E_{\text {ad }}\right)$ and fluorescence energies $\left(E_{\mathrm{ff}}\right)$ as well as corresponding Stoke's shifts $(\lambda)[\mathrm{eV}]$ for ADMM-PBE0 and sTDA kernels for the first excited singlet state for a series of fluorescent COFs.

\begin{tabular}{|c|c|c|c|c|c|c|c|c|}
\hline & \multicolumn{2}{|c|}{$E_{\text {vert }}$} & \multicolumn{2}{c|}{$E_{\text {ad }}$} & \multicolumn{2}{c|}{$E_{\mathrm{f}}$} & \multicolumn{2}{c|}{$\lambda$} \\
\cline { 2 - 9 } & ADMM & sTDA & ADMM & sTDA & ADMM & sTDA & ADMM & sTDA \\
\hline $05000 \mathrm{~N} 2$ & 4.22 & 4.18 & 4.12 & 3.82 & 4.00 & 3.37 & 0.22 & 0.06 \\
$05001 \mathrm{~N} 2$ & 2.91 & 2.31 & 2.68 & 2.13 & 2.42 & 1.67 & 0.49 & 0.64 \\
$15100 \mathrm{~N} 2$ & 2.42 & 1.98 & 2.56 & 2.10 & 1.43 & 0.96 & 0.99 & 1.02 \\
$15101 \mathrm{~N} 2$ & 2.17 & 1.69 & 2.06 & 1.50 & 1.25 & 0.70 & 0.92 & 0.99 \\
$20610 \mathrm{~N} 2$ & 2.97 & 2.74 & 2.75 & 2.46 & 2.14 & 1.82 & 0.83 & 0.92 \\
$20611 \mathrm{~N} 2$ & 2.56 & 2.25 & 2.44 & 2.07 & 2.01 & 1.38 & 0.55 & 0.87 \\
\hline MAD & \multicolumn{2}{|c|}{0.35} & \multicolumn{2}{c|}{0.42} & \multicolumn{2}{c|}{0.31} & \multicolumn{2}{c|}{0.13} \\
\hline
\end{tabular}

\section{Conclusions}

Approaches based on TDDFT are well established to calculate excited state properties. However, when dealing with periodic systems, the routine usage of hybrid functionals is often hindered due to the increased computational costs in comparison to GGA functionals. We presented two approximate schemes based on the ADMM and sTDA approaches with the goal to reach hybrid functional accuracy at reduced cost. Benchmark results for molecular and periodic systems show that the two methods can indeed reach the required accuracy for excited-state properties and allow for an efficient calculation of many excited states. First, the implemented ADMM-approximated hybrid functional kernel leads to a speed-up of at least one order of magnitude in comparison to conventional hybrid functional TDDFT and thus enables practical calculations on large systems of hundreds of atoms at moderate cost. Timings could be further improved by accelerating the evaluation of the exact exchange integrals using either standard resolution-of-the-identity approaches or semi-numerical integration. In comparison to inherent methodological errors of TDDFT due to the chosen density functional, the loss in accuracy for ADMM-approximated kernels is minor when choosing auxiliary basis sets of at least triple-zeta size. ADMM / TZVP +tzp MADs in excited-state bond lengths are in the range of $0.2 \mathrm{pm}$ and corresponding vertical and adiabatic excitation and fluorescence energies are off by 0.02 to $0.04 \mathrm{eV}$. In contrast, conventional PBE0 / TZVP bond lengths and adiabatic excitation energies show MADs of $1.0 \mathrm{pm}$ and 0.4 $\mathrm{eV}$ with respect to EOM-CCSD / def2-TZVPP reference data. The given ADMM accuracy estimate represents a lower limit that was found to be reliable when choosing the simplest ADMM variant based on basis projection in combination with a triple-zeta auxiliary basis. It could be further improved by either adding an exchange density functional correction to compensate the incompleteness error or - regarding the perpendicular methodological error 
- by switching to more sophisticated ADMM schemes with the latter however increasing the complexity of implementation. The semi-empirical sTDA method is particularly useful when aiming for broad-band absorption or emission spectra and efficient pre-screening of large-scale test sets. An order of magnitude speedup was achieved with sTDA in comparison to ADMM when computing spectra over the lowest 500 excitation energies, with resulting timings being in the range of minutes rather than hours. Due to the reduced amount of ERIs, the sTDA kernel also accelerates timings by a factor of two for each ES geometry optimization cycle. However, an increased number of optimization steps can lead to total timings comparable to ADMM. We expect that further savings could be achieved when optimizing the current sTDA ES gradient implementation as well as when choosing a semi-empirical GS reference. In comparison to hybrid functional kernels, sTDA MADs in excited-state bond lengths with respect to EOM-CCSD reference results are only slightly increased to $1.1 \mathrm{pm}$. According to the molecular benchmarks, the error estimate for vertical and adiabatic excitation and fluorescence energies can be assumed to be below $0.5 \mathrm{eV}$, a deviation which is slightly increased, but still comparable to the MAD of $0.4 \mathrm{eV}$ of conventional PBE0. Similar relative error ranges are found for the investigated COFs and a comparison of ADMM and sTDA oscillator strengths suggests qualitative accuracy of the tight binding method. The most critical parameter in the sTDA method is the exact exchange scaling. This parameter should be adjusted with respect to the selected hybrid functional ground-state reference and a sub-optimal value can lead to large geometrical errors and even negative excitation energies. In the case of Formylchloride, the scaling parameter had to be adjusted to avoid dissociation when optimizing the first excited singlet state. Further studies are required to investigate the optimal fraction of exact exchange for periodic systems since the choice might differ from the values that were originally suggested for molecular systems. Further empirical shifts might also be required when combining the sTDA excited-state method with corresponding semiempirical ground-state calculations. A consistent setup which remains to be investigated but which we expect to pave the way to treat even larger systems. It also remains to be investigated in future work if efficiency and accuracy of ADMM-approximated hybrid functional TDDFT and sTDA are beneficial for excited-state molecular dynamic simulations.

\section{Acknowledgement}

This work was supported in parts by the MARVEL National Centre for Competency in Research funded by the Swiss National Science Foundation (grant agreement ID 51NF40182892). We thank the Swiss National Supercomputing Center (CSCS) for providing com-

putational resources (Grant No. S1109). Furthermore this work has received funding from 
the European Union's Horizon 2020 research and innovation programme under the Marie Skłodowska-Curie grant agreement no. 798196.

\section{References}

(1) González, L.; Escudero, D.; Serrano-Andrés, L. Progress and Challenges in the Calculation of Electronic Excited States. ChemPhysChem 2012, 13, 28-51.

(2) Dreuw, A.; Head-Gordon, M. Single-Reference ab Initio Methods for the Calculation of Excited States of Large Molecules. Chem. Rev. 2005, 105, 4009-4037.

(3) Laurent, A. D.; Jacquemin, D. TD-DFT benchmarks: A review. Int. J. Quantum Chem. 2013, 113, 2019-2039.

(4) de Wergifosse, M.; Grimme, S. Perspective on Simplified Quantum Chemistry Methods for Excited States and Response Properties. J. Phys. Chem. A 2021, 125, 3841-3851.

(5) Grimme, S.; Schreiner, P. R. Computational Chemistry: The Fate of Current Methods and Future Challenges. Angew. Chem. Int. Ed. 2018, 5\%, 4170-4176.

(6) Dierksen, M.; Grimme, S. The Vibronic Structure of Electronic Absorption Spectra of Large Molecules: A Time-Dependent Density Functional Study on the Influence of Exact Hartree-Fock Exchange. J. Phys. Chem. A 2004, 108, 10225-10237.

(7) Merlot, P.; Izsák, R.; Borgoo, A.; Kjærgaard, T.; Helgaker, T.; Reine, S. Chargeconstrained auxiliary-density-matrix methods for the Hartree-Fock exchange contribution. J. Chem. Phys. 2014, 141, 094104.

(8) Guidon, M.; Hutter, J.; VandeVondele, J. Auxiliary Density Matrix Methods for Hartree-Fock Exchange Calculations. J. Chem. Theory Comput. 2010, 6, 2348-2364.

(9) Kumar, C.; Fliegl, H.; Jensen, F.; Teale, A. M.; Reine, S.; Kjærgaard, T. Accelerating Kohn-Sham response theory using density fitting and the auxiliary-density-matrix method. Int. J. Quantum Chem. 2018, 118, e25639.

(10) Rebolini, E.; Izsák, R.; Reine, S. S.; Helgaker, T.; Pedersen, T. B. Comparison of Three Efficient Approximate Exact-Exchange Algorithms: The Chain-of-Spheres Algorithm, Pair-Atomic Resolution-of-the-Identity Method, and Auxiliary Density Matrix Method. J. Chem. Theory Comput. 2016, 12, 3514-3522. 
(11) Merlot, P.; Kjærgaard, T.; Helgaker, T.; Lindh, R.; Aquilante, F.; Reine, S.; Pedersen, T. B. Attractive electron-electron interactions within robust local fitting approximations. J. Comput. Chem. 2013, 34, 1486-1496.

(12) Manzer, S. F.; Epifanovsky, E.; Head-Gordon, M. Efficient Implementation of the Pair Atomic Resolution of the Identity Approximation for Exact Exchange for Hybrid and Range-Separated Density Functionals. J. Chem. Theory Comput. 2015, 11, 518-527.

(13) Neese, F.; Wennmohs, F.; Hansen, A.; Becker, U. Efficient, approximate and parallel Hartree-Fock and hybrid DFT calculations. A 'chain-of-spheres' algorithm for the Hartree-Fock exchange. Chem. Phys. 2009, 356, 98-109.

(14) Izsák, R.; Neese, F. An overlap fitted chain of spheres exchange method. J. Chem. Phys. 2011, 135, 144105.

(15) Laqua, H.; Thompson, T. H.; Kussmann, J.; Ochsenfeld, C. Highly Efficient, LinearScaling Seminumerical Exact-Exchange Method for Graphic Processing Units. J. Chem. Theory Comput. 2020, 16, 1456-1468.

(16) Holzer, C. An improved seminumerical Coulomb and exchange algorithm for properties and excited states in modern density functional theory. J. Chem. Phys. 2020, 153, 184115 .

(17) Ohno, K. Some remarks on the Pariser-Parr-Pople method. Theor. Chim. Acta 1964, 2, 219-227.

(18) Klopman, G. A Semiempirical Treatment of molecular Structures. II. Molecular Terms and Application to diatomic Molecules. J. Am. Chem. Soc. 1964, 86, 4550-4557.

(19) Nishimoto, K.; Mataga, N. Z. Phys. Chem. 1957, 12, 335.

(20) Bannwarth, C.; Grimme, S. A simplified time-dependent density functional theory approach for electronic ultraviolet and circular dichroism spectra of very large molecules. Comput. Theor. Chem. 2014, 1040-1041, 45 - 53.

(21) Grimme, S. A simplified Tamm-Dancoff density functional approach for the electronic excitation spectra of very large molecules. J. Chem. Phys. 2013, 138, 244104.

(22) Grimme, S.; Bannwarth, C. Ultra-fast computation of electronic spectra for large systems by tight-binding based simplified Tamm-Dancoff approximation (sTDA-xTB). J. Chem. Phys. 2016, 145, 054103. 
(23) de Wergifosse, M.; Bannwarth, C.; Grimme, S. A Simplified Spin-Flip Time-Dependent Density Functional Theory Approach for the Electronic Excitation Spectra of Very Large Diradicals. J. Phys. Chem. A 2019, 123, 5815-5825.

(24) De Wergifosse, M.; Grimme, S. Nonlinear-response properties in a simplified timedependent density functional theory (sTD-DFT) framework: Evaluation of excitedstate absorption spectra. J. Chem. Phys. 2019, 150, 094112.

(25) De Wergifosse, M.; Grimme, S. Nonlinear-response properties in a simplified timedependent density functional theory (sTD-DFT) framework: Evaluation of the first hyperpolarizability. J. Chem. Phys. 2018, 149, 024108.

(26) Bannwarth, C.; Seibert, J.; Grimme, S. Electronic Circular Dichroism of [16]Helicene With Simplified TD-DFT: Beyond the Single Structure Approach. Chirality 2016, 28, 365-369.

(27) Risthaus, T.; Hansen, A.; Grimme, S. Excited states using the simplified TammDancoff-Approach for range-separated hybrid density functionals: development and application. Phys. Chem. Chem. Phys. 2014, 16, 14408-14419.

(28) Elstner, M.; Porezag, D.; Jungnickel, G.; Elsner, J.; Haugk, M.; Frauenheim, T.; Suhai, S.; Seifert, G. Self-consistent-charge density-functional tight-binding method for simulations of complex materials properties. Phys. Rev. B 1998, 58, 7260-7268.

(29) Seibert, J.; Pisarek, J.; Schmitz, S.; Bannwarth, C.; Grimme, S. Extension of the element parameter set for ultra-fast excitation spectra calculation (sTDA-xTB). Mol. Phys. 2019, 117, 1104-1116.

(30) Cho, Y.; Bintrim, S. J.; Berkelbach, T. C. A simplified GW/BSE approach for charged and neutral excitation energies of large molecules and nanomaterials. 2021; arXiv:2109.04421.

(31) Rüger, R.; van Lenthe, E.; Heine, T.; Visscher, L. Tight-binding approximations to time-dependent density functional theory - A fast approach for the calculation of electronically excited states. J. Chem. Phys. 2016, 144, 184103.

(32) Asadi-Aghbolaghi, N.; Pototschnig, J.; Jamshidi, Z.; Visscher, L. Effects of ligands on (de-)enhancement of plasmonic excitations of silver, gold and bimetallic nanoclusters: TD-DFT + TB calculations. Phys. Chem. Chem. Phys. 2021, 23, 17929-17938. 
(33) Van Caillie, C.; Amos, R. D. Geometric derivatives of excitation energies using SCF and DFT. Chem. Phys. Lett. 1999, 308, 249-255.

(34) Van Caillie, C.; Amos, R. D. Geometric derivatives of density functional theory excitation energies using gradient-corrected functionals. Chem. Phys. Lett. 2000, 317, 159-164.

(35) Furche, F.; Ahlrichs, R. Adiabatic time-dependent density functional methods for excited state properties. J. Chem. Phys. 2002, 117, 7433-7447.

(36) Hutter, J. Excited state nuclear forces from the Tamm-Dancoff approximation to timedependent density functional theory within the plane wave basis set framework. $J$. Chem. Phys. 2003, 118, 3928-3934.

(37) Petrenko, T.; Kossmann, S.; Neese, F. Efficient time-dependent density functional theory approximations for hybrid density functionals: Analytical gradients and parallelization. J. Chem. Phys. 2011, 134, 054116.

(38) Grotjahn, R.; Furche, F.; Kaupp, M. Development and Implementation of ExcitedState Gradients for Local Hybrid Functionals. J. Chem. Theory Comput. 2019, 15, 5508-5522.

(39) Kretz, B.; Egger, D. A. Accurate Molecular Geometries in Complex Excited-State Potential Energy Surfaces from Time-Dependent Density Functional Theory. J. Chem. Theory Comput. 2021, 17, 357-366.

(40) Sokolov, M.; Bold, B. M.; Kranz, J. J.; Höfener, S.; Niehaus, T. A.; Elstner, M. Analytical Time-Dependent Long-Range Corrected Density Functional Tight Binding (TDLC-DFTB) Gradients in DFTB+: Implementation and Benchmark for Excited-State Geometries and Transition Energies. J. Chem. Theory Comput. 2021, 17, 2266-2282.

(41) Kühne, T. D.; Iannuzzi, M.; Del Ben, M.; Rybkin, V. V.; Seewald, P.; Stein, F.; Laino, T.; Khaliullin, R. Z.; Schütt, O.; Schiffmann, F.; Golze, D.; Wilhelm, J.; Chulkov, S.; Bani-Hashemian, M. H.; Weber, V.; Borštnik, U.; Taillefumier, M.; Jakobovits, A. S.; Lazzaro, A.; Pabst, H.; Müller, T.; Schade, R.; Guidon, M.; Andermatt, S.; Holmberg, N.; Schenter, G. K.; Hehn, A.; Bussy, A.; Belleflamme, F.; Tabacchi, G.; Glöß, A.; Lass, M.; Bethune, I.; Mundy, C. J.; Plessl, C.; Watkins, M.; VandeVondele, J.; Krack, M.; Hutter, J. CP2K: An electronic structure and molecular dynamics software package - Quickstep: Efficient and accurate electronic structure calculations. J. Chem. Phys. 2020, 152, 194103. 
(42) VandeVondele, J.; Krack, M.; Mohamed, F.; Parrinello, M.; Chassaing, T.; Hutter, J. Quickstep: Fast and accurate density functional calculations using a mixed Gaussian and plane waves approach. Comput. Phys. Commun. 2005, 167, 103-128.

(43) Lippert, G.; Hutter, J.; Parrinello, M. A hybrid Gaussian and plane wave density functional scheme. Mol. Phys. 1997, 92, 477-488.

(44) Iannuzzi, M.; Chassaing, T.; Wallman, T.; Hutter, J. Ground and Excited State Density Functional Calculations with the Gaussian and Augmented-Plane-Wave Method. CHIMIA Int. J. Chem. 2005, 59, 499-503.

(45) Strand, J.; Chulkov, S. K.; Watkins, M. B.; Shluger, A. L. First principles calculations of optical properties for oxygen vacancies in binary metal oxides. J. Chem. Phys. 2019, 150, 044702.

(46) Poli, E.; Elliott, J. D.; Chulkov, S. K.; Watkins, M. B.; Teobaldi, G. The Role of Cation-Vacancies for the Electronic and Optical Properties of Aluminosilicate Imogolite Nanotubes: A Non-local, Linear-Response TDDFT Study. Front. Chem. 2019, 7, 210.

(47) Budzak, S.; Scalmani, G.; Jacquemin, D. Accurate Excited-State Geometries: A CASPT2 and Coupled-Cluster Reference Database for Small Molecules. J. Chem. Theory Comput. 2017, 13, 6237-6252.

(48) Ongari, D.; Yakutovich, A. V.; Talirz, L.; Smit, B. Building a Consistent and Reproducible Database for Adsorption Evaluation in Covalent-Organic Frameworks. ACS Cent. Sci. 2019, 5, 1663-1675.

(49) Ongari, D.; Talirz, L.; Smit, B. Too Many Materials and Too Many Applications: An Experimental Problem Waiting for a Computational Solution. ACS Cent. Sci. 2020, $6,1890-1900$.

(50) Hirata, S.; Head-Gordon, M. Time-dependent density functional theory within the Tamm-Dancoff approximation. Chem. Phys. Lett. 1999, 314, 291-299.

(51) Casida, M. E. In Recent Advances in Density Functional Methods, Part I; Chong, D. P., Ed.; Singapore, World Scientific, 1995, pp. 155.

(52) Guidon, M.; Hutter, J.; VandeVondele, J. Auxiliary Density Matrix Methods for Hartree-Fock Exchange Calculations. J. Chem. Theory Comput. 2010, 6, 2348-2364. 
(53) Ghosh, D. C.; Islam, N. Whether electronegativity and hardness are manifest two different descriptors of the one and the same fundamental property of atoms? @ TA quest. Int. J. Quantum Chem. 2011, 111, 40-51.

(54) Marx, D.; Hutter, J. In Modern Methods and Algorithms of Quantum Chemistry; Grotendorst, J., Ed.; John von Neumann Institute for Computing, Forschungszentrum Jülich, Jülich, 2000, pp. 301-449 (first edition, paperback, ISBN 3-00005618-1) or pp. 329-477 (second edition, hardcover, ISBN 3-00-005834-6), see http://www.theochem.rub.de/go/cprev.html.

(55) Essmann, U.; Perera, L.; Berkowitz, M. L.; Darden, T.; Lee, H.; Pedersen, L. G. A smooth particle mesh Ewald method. J. Chem. Phys. 1995, 103, 8577-8593.

(56) Furche, F.; Ahlrichs, R. Erratum: "Time-dependent density functional methods for excited state properties" [J. Chem. Phys. 117, 7433 (2002)]. J. Chem. Phys. 2004, 121, $12772-12773$.

(57) Mayhall, N. J.; Raghavachari, K.; Hratchian, H. P. ONIOM-based QM:QM electronic embedding method using Löwdin atomic charges: Energies and analytic gradients. J. Chem. Phys. 2010, 132, 114107.

(58) Gaussian 16, M. J. F.; Trucks, G. W.; Schlegel, H. B.; Scuseria, G. E.; Robb, M. A.; Cheeseman, J. R.; Scalmani, G.; Barone, V.; Petersson, G. A.; Nakatsuji, H.; Li, X.; Caricato, M.; Marenich, A. V.; Bloino, J.; Janesko, B. G.; Gomperts, R.; Mennucci, B.; Hratchian, H. P.; Ortiz, J. V.; Izmaylov, A. F.; Sonnenberg, J. L.; WilliamsYoung, D.; Ding, F.; Lipparini, F.; Egidi, F.; Goings, J.; Peng, B.; Petrone, A.; Henderson, T.; Ranasinghe, D.; Zakrzewski, V. G.; Gao, J.; Rega, N.; Zheng, G.; Liang, W.; Hada, M.; Ehara, M.; Toyota, K.; Fukuda, R.; Hasegawa, J.; Ishida, M.; Nakajima, T.; Honda, Y.; Kitao, O.; Nakai, H.; Vreven, T.; Throssell, K.; Montgomery, J. A., Jr.; Peralta, J. E.; Ogliaro, F.; Bearpark, M. J.; Heyd, J. J.; Brothers, E. N.; Kudin, K. N.; Staroverov, V. N.; Keith, T. A.; Kobayashi, R.; Normand, J.; Raghavachari, K.; Rendell, A. P.; Burant, J. C.; Iyengar, S. S.; Tomasi, J.; Cossi, M.; Millam, J. M.; Klene, M.; Adamo, C.; Cammi, R.; Ochterski, J. W.; Martin, R. L.; Morokuma, K.; Farkas, O.; Foresman, J. B.; Fox, D. J. Revision C.01, Gaussian Inc., Wallingford CT, 2016.

(59) Weigend, F.; Ahlrichs, R. Balanced basis sets of split valence, triple zeta valence and quadruple zeta valence quality for $\mathrm{H}$ to $\mathrm{Rn}$ : Design and assessment of accuracy. Phys. Chem. Chem. Phys. 2005, \%, 3297. 
(60) Balasubramani, S. G.; Chen, G. P.; Coriani, S.; Diedenhofen, M.; Frank, M. S.; Franzke, Y. J.; Furche, F.; Grotjahn, R.; Harding, M. E.; Hättig, C.; Hellweg, A.; Helmich-Paris, B.; Holzer, C.; Huniar, U.; Kaupp, M.; Marefat Khah, A.; Karbalaei Khani, S.; Müller, h.; Mack, F.; Nguyen, B. D.; Parker, S. M.; Perlt, E.; Rappoport, D.; Reiter, K.; Roy, S.; Rückert, M.; Schmitz, G.; Sierka, M.; Tapavicza, E.; Tew, D. P.; van Wüllen, C.; Voora, V. K.; Weigend, F.; Wodyński, A.; Yu, J. M. TURBOMOLE: Modular program suite for ab initio quantum-chemical and condensedmatter simulations. J. Chem. Phys. 2020, 152, 184107.

(61) Weigend, F.; Furche, F.; Ahlrichs, R. Gaussian basis sets of quadruple zeta valence quality for atoms H-Kr. J. Chem. Phys. 2003, 119, 12753-12762.

(62) VandeVondele, J.; Hutter, J. Gaussian basis sets for accurate calculations on molecular systems in gas and condensed phases. J. Chem. Phys. 2007, 127, 114105.

(63) Fischer, G.; Ross, I. G. Electronic Spectrum of Dicyanoacetylene. 1. Calculations of the Geometries and Vibrations of Ground and Excited States of Diacetylene, Cyanoacetylene, Cyanogen, Triacetylene, Cyanodiacetylene, and Dicyanoacetylene. J. Phys. Chem. A 2003, 107, 10631-10636.

(64) Brémond, E.; Savarese, M.; Adamo, C.; Jacquemin, D. Accuracy of TD-DFT Geometries: A Fresh Look. J. Chem. Theory Comput. 2018, 14, 3715-3727.

(65) Fang, C.; Oruganti, B.; Durbeej, B. How Method-Dependent Are Calculated Differences between Vertical, Adiabatic, and 0-0 Excitation Energies? J. Phys. Chem. A 2014, $118,4157-4171$.

(66) Peach, M. J. G.; Benfield, P.; Helgaker, T.; Tozer, D. J. Excitation energies in density functional theory: An evaluation and a diagnostic test. J. Chem. Phys. 2008, 128, 044118 .

(67) Send, R.; Kühn, M.; Furche, F. Assessing Excited State Methods by Adiabatic Excitation Energies. J. Chem. Theory Comput. 2011, 7, 2376-2386.

(68) Côté, A. P.; Benin, A. I.; Ockwig, N. W.; O’Keeffe, M.; Matzger, A. J.; Yaghi, O. M. Porous, Crystalline, Covalent Organic Frameworks. Science 2005, 310, 1166-1170.

(69) Das, G.; Biswal, B. P.; Kandambeth, S.; Venkatesh, V.; Kaur, G.; Addicoat, M.; Heine, T.; Verma, S.; Banerjee, R. Chemical sensing in two dimensional porous covalent organic nanosheets. Chem. Sci. 2015, 6, 3931-3939. 
(70) Das, P.; Chakraborty, G.; Tyagi, S.; Mandal, S. K. Design of Fluorescent and Robust Covalent Organic Framework Host Matrices for Illuminating Mechanistic Insight into Solvatochromic Decoding. ACS Appl. Mater. Interfaces 2020, 12, 52527-52537. 